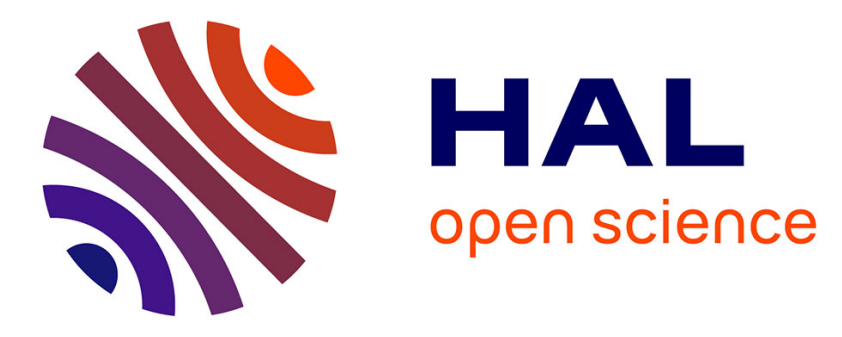

\title{
La construction processionnelle de l'espace communautaire
}

\author{
Ludolf Kuchenbuch, Joseph Morsel, Dieter Scheler
}

\section{To cite this version:}

Ludolf Kuchenbuch, Joseph Morsel, Dieter Scheler. La construction processionnelle de l'espace communautaire. Didier Boisseuil, Pierre Chastang, Laurent Feller et Joseph Morsel. Écritures de l'espace social. Mélanges d'histoire médiévale offerts à Monique Bourin, Publications de la Sorbonne, pp.139182, 2010, Histoire ancienne et médiévale - 101. halshs-00825504

\section{HAL Id: halshs-00825504 https://shs.hal.science/halshs-00825504}

Submitted on 28 May 2013

HAL is a multi-disciplinary open access archive for the deposit and dissemination of scientific research documents, whether they are published or not. The documents may come from teaching and research institutions in France or abroad, or from public or private research centers.
L'archive ouverte pluridisciplinaire HAL, est destinée au dépôt et à la diffusion de documents scientifiques de niveau recherche, publiés ou non, émanant des établissements d'enseignement et de recherche français ou étrangers, des laboratoires publics ou privés. 
Histoire ancienne et médiévale - 101

Université Paris 1 Panthéon-Sorbonne

\title{
Écritures de l'espace social
}

\author{
Mélanges d'histoire médiévale \\ offerts à Monique Bourin
}

Travaux réunis par

D. Boisseuil, P. Chastang, L. Feller et J. Morsel

\author{
Ouvrage publié avec le concours \\ du Conseil scientifique de l'Université Paris 1 \\ et du Laboratoire de médiévistique occidentale de Paris (Lamop)
}


Couverture : Modène, Estense, Lat. 209, f. 12 : Leonardo Dati, De sphaera, enluminé vers 1460-1470 à Ferrare, par Cristoforo de Predis.

Composition typographique : Laurent Tournier

Version tirée à part, corrigée et restaurée en fonction du manuscrit original

(C) Publications de la Sorbonne, 2010, et les auteurs

212, rue Saint-Jacques, 75005 Paris

www.univ-paris1.fr - publisor@univ-paris1.fr

ISBN : 978-2-85944-654-3

ISSN : 0290-4500

Les opinions exprimées dans cet ouvrage n'engagent que leurs auteurs

" Aux termes du Code de la propriété intellectuelle, toute reproduction ou représentation, intégrale ou partielle, de la présente publication, faite par quelque procédé que ce soit (reprographie, microfilmage, scannérisation, numérisation...) sans le consentement de l'auteur ou de ses ayants droit ou ayants cause est illicite et constitue une contrefaçon sanctionnée par les articles L.335-2 et suivants du Code de la propriété intellectuelle. II est rappelé également que l'usage abusif et collectif de la photocopie met en danger l'équilibre économique des circuits du livre. » 


\title{
La construction processionnelle de l'espace communautaire
}

\author{
Ludolf Kuchenbuch, Joseph Morsel, Dieter Scheler
}

\begin{abstract}
On peut affirmer que l'État, en tant que tel, est né d'une sorte de fusion «chimique» entre l'éleveur et le cultivateur, une fois que l'on se rendit compte qu'il était possible d'appliquer les techniques de coercition animale à une masse paysanne inerte. En dehors de leur rôle de «maitres des eaux fertilisantes ", les premiers dictateurs se dénommaient eux-mêmes «bergers du peuple». Les masses doivent être rassemblées, exploitées, parquées (pour les protéger des «loups » humains venus de l'extérieur) et, si besoin est, conduites à l'abattoir.
\end{abstract}

Bruce Chatwin, The Songlines, 1987

« Ce sont les hommes qui vont à la messe, pas les parcelles », a fait un jour observer Monique Bourin ${ }^{1}$. Au-delà de son apparent caractère d'évidence, cette boutade soulève le problème des multiples raccourcis qu'opèrent les historiens lorsqu'ils traitent des rapports des hommes aux terres. De même que parler d'échanges, de transfert ou de circulation des terres montre à l'évidence une confusion entre le substrat sur lequel repose la transaction et l'objet de la transaction, à savoir des droits d'usage plus ou moins exclusif (ce que nous appelons "droit de propriété ») sur certains lieux² et qui, eux, font circuler les hommes, lorsque les hommes vont à la messe, ils réalisent la pa-

\footnotetext{
1. Au cours d'un séminaire de recherche à Tours, le 30 juin 2000 (déclaration rapportée par S. Leturcq, «Territoires agraires et limites paroissiales », Médiévales, 49 (2005), p. 89-104, ici p. 92-93).

2. Ce sont donc les hommes qui circulent, et non pas les terres. Que les rapports entre hommes soient ainsi convertis en rapports entre terres, ou plus exactement inversés (comme si c'était entre les hommes que circulaient les terres alors qu'en réalité c'est entre les terres que circulent les hommes - inversion sans doute de même nature que celle qu'avait signalée Marx en disant que c'est la terre qui hérite du fils aîné et non pas l'inverse) ne devrait-il pas être considéré comme une manière de masquer l'enjeu du problème, à savoir celui de la mobilité de la maind'œuvre?
} 
roisse - réalisation locale et représentation de l'ecclesia tout entière - et non pas la communauté d'exploitation des parcelles en quoi consiste la communauté d'habitants. C'est ce problème de l'articulation de la paroisse et de la communauté d'habitants que nous voulons aborder ici, en donnant raison à Monique Bourin quant à la nécessité de bien distinguer les deux niveaux d'organisation sociale, non parce que l'un serait religieux et l'autre économique, mais parce qu'ils renvoient à des modes d'organisation spatiale radicalement différents.

La question de la formation médiévale des espaces de domination (les territoires) et du rôle spécifique qu'ont pu jouer l'église, le château, le droit ou la coutume, voire les conditions "naturelles » (relief, pédologie, épidémies, etc.), préoccupe une partie notable des médiévistes depuis les années 1970 et a donné naissance à divers modèles explicatifs de grande valeur heuristique (incastellamento, encellulement, communalisme, ensagrerament, inecclesiamento...). Loin de vouloir ajouter un nouveau modèle à cette liste, quelques médiévistes initialement groupés autour de Monique Bourin ont entrepris, à partir de 2002, de revisiter ce processus en insistant sur son aspect spatial ${ }^{3}$, c'està-dire en tant que processus de sociogenèse non pas dans l'espace, mais de l'espace - bref en considérant l'espace non pas comme un cadre ou comme une ressource, mais comme un résultat, sous la forme d'un ensemble de représentations, discours et pratiques, en dehors desquels l'espace n'existe pas 4 .

3. Le fil d'Ariane de ce projet a été constitué par les concepts, faussement transparents, d' « habitant » et " habiter », signalant non pas le "simple » fait d'avoir un toit sur la tête ou une adresse, mais un certain rapport socio-spatial, à la fois aux lieux et aux autres personnes présentes localement. C'est ainsi la modification de ce mode d'inscription dans l'espace, engendrant ce que certains géographes appellent « cospatialité » au lieu de la juxtaposition antérieure d'exofocalisations (les homines éventuellement groupés en un lieu étant focalisés chacun sur un pôle spécifique et extérieur, la villa du maître, parfois fort éloignée : cf. J. Demade, « Les „corvées" en Haute-Allemagne. Du rapport de production au symbole de domination ( $\mathrm{XI}^{\mathrm{e}}$ XIVe siècles) », dans Pour une anthropologie du prélèvement seigneurial dans les campagnes médiévales (XI'-XIVe siècles), M. Bourin, P. Martinez Sopena dir., Paris, Publications de la Sorbonne, 2004, p. 337-364, ici p. 344-345, ou encore J.-P. Devroey, "Communiquer et signifier entre seigneurs et paysans ", dans Comunicare e significare nell'alto medioevo, Spolète, Centro italiano di studi sull'Alto Medioevo (Settimane di studi..., 52), 2005, p. 121-152, ici p. 137-141) qu'entendait examiner ce projet d'étude de «La formation médiévale des communautés d'habitants ", dont le " coup d'envoi » a été le colloque organisé à Xanten en juin 2003 par les trois auteurs de ce texte, et dont certains résultats sont consultables en ligne (http://lamop.univ-paris1.fr/lamop/LAMOP/Xanten/Xanten.htm et http://lamop.univparis1.fr/lamop/LAMOP/Gif/Habiter.htm).

4. Ce n'est pas ici le lieu de développer ces points. Nous nous contenterons de renvoyer d'une part à l'ouvrage clé de H. Lefebvre, La production de l'espace, Paris, Anthropos, 1974 ; aux observations critiques d'A. Guerreau, "Quelques caractères spécifiques de l'espace féodal européen ", dans L'État ou le roi. Les fondations de la modernité monarchique en France (XIVe-XVII siècles), N. Bulst, R. Descimon, A. Guerreau dir., Paris, Éditions de la MSH, 1996, p. 85-101, et surtout "Il significato dei luoghi nell'Occidente medievale : struttura e dinamica di uno 'spazio' speci- 
Deux distinctions structurelles sous-tendent ce projet : d'une part la distinction entre organisation sociale fondée sur la parenté et organisation sociale fondée sur l'espace, si bien que la spatialisation de l'organisation sociale n'aurait pu qu'être corrélative à une « déparentalisation » du social5. La seconde distinction structurelle consiste à admettre que la paroisse et la communauté d'habitants constituent initialement deux formes d'organisation sociale aux natures profondément distinctes - au point qu'il soit difficile voire impossible de parler pour le Moyen Âge (ou en tout cas l'essentiel de la période médiévale) de " communauté paroissiale » proprement dite 6 .

Cette seconde distinction repose sur le fait que la paroisse se définit comme un ensemble de rapports interpersonnels (sacramentels et matériels) entre le curé et chaque paroissien, tous les liens entre paroissiens étant tendanciellement médiatisés par le curé - alors que la communauté d'habitants se définit plutôt comme un ensemble de rapports entre feux interdépendants du point de vue productif, fiscal et défensif. De ce fait, la paroisse se définit par rapport à son centre (l'église et le cimetière) et non par rapport à ses limites7, alors que la communauté d'habitants se définit par rapport à ses limites

fico », dans Arti e storia nel Medioevo, I : Tempi - Spazi - Istituzioni, E. Castelnuovo, G. Sergi (dir.), Turin, Einaudi, 2002, p. 201-239; enfin aux bilans croisés histoire/archéologie de M. Bourin, É. Zadora-Rio, "L'espace » dans Les tendances actuelles de l'histoire du Moyen Âge en France et en Allemagne, O.G. Oexle, J.-C. Schmitt dir., Paris, Publications de la Sorbonne, 2002 p. 493-510, et « Pratiques de l'espace : les apports comparés des données textuelles et des données archéologiques " dans Construction de l'espace au Moyen Âge : pratiques et représentations. XXXVII congrès de la S.H.M.E.S. (Mulhouse, 2-4 juin 2006), Paris, Publications de la Sorbonne, 2007, p. 39-55. Cf. également infra, n. 117.

5. Là encore, on ne peut que mentionner ici cet aspect, qui a fait l'objet de diverses discussions depuis la publication de l'essai de J. Morsel, L'Histoire (du Moyen Âge) est un sport de combat... Réflexions sur les finalités de l'Histoire du Moyen Âge destinées à une société dans laquelle même les étudiants d'Histoire s'interrogent, http://lamop.univ-paris1.fr/W3/JosephMorsel/index.htm (2007).

6. Cf. J. Morsel, « Habiter la ville. Remarques sur le problème de l'appartenance urbaine médiévale (XII ${ }^{\mathrm{e}} \mathrm{XIII}{ }^{\mathrm{e}}$ siècle) », à paraître.

7. Cf. les diverses contributions qui composent le dossier « Formation et transformations des territoires paroissiaux » publié sous la direction de D. Iogna-Prat et É. Zadora-Rio, Médiévales, 49 (2005), p. 5-119, qui signalent le caractère tardif et non systématique des délimitations, malgré l'apparition (elle aussi tardive) d'un discours canonique signalant les limites parmi les critères de définition de la paroisse. Toutefois, il apparait clairement que ce qui a motivé la précision des limites, lorsqu'il y en a, est la nécessité de délimiter les droits seigneuriaux concurrents s'exerçant sur les paroisses, qu'il s'agisse de la dîme, de la dotation ou du droit de présentation (c'est aussi ce qu'observe en Angleterre S. Hindle, "Beating the Bounds of the Parish : Order, Memory and Identity in the English Local Community, c. 1500-1700 ", dans Defining Community in Early Modern Europe, M.J. Halvorson, K.E. Spierling dir., Aldershot, Ashgate, 2008, p. 205-227, qui signale (p. 217, 220) à la fois le caractère tardif - dernier quart du $\mathrm{XVI}^{\mathrm{e}}$ siècle - des descriptions écrites ou figurées des limites paroissiales et le lien entre ces descriptions et le souci de préserver les droits, privilèges, revenus et dîmes. Je tiens à remercier Kouky Fianu de m'avoir signalé et procuré ce travail). Cf. également la contribution de 
(d'où la fréquence de la représentation métonymique des murs sur les sceaux urbains) et non par rapport à son centre (la plupart du temps inexistant en tant que tel : le cas des beffrois est exceptionnel). Bref, la paroisse est un ensemble strictement interpersonnel et polarisé, alors que la communauté d'habitants apparait comme un ensemble « interfocal » défini par rapport à un lieu démarqué8.

Pourtant, les modernistes admettent, à la suite des textes qu'ils utilisent, l'existence de telles « communautés paroissiales »- au point que les paroisses ont de fait fini par constituer des espaces délimités et que les limites paroissiales ont pu être en divers endroits converties en limites communales au tournant de l'époque contemporaine ${ }^{9}$. On doit alors s'interroger sur les modalités pratiques qui ont conduit à faire coïncider socialement (et pas seulement de fait, par simple et éventuelle coextensivité) communauté d'habitants et paroisse. Une pratique sociale récurrente et institutionnalisée semblerait pouvoir être examinée sous cet angle : les processions générales, qui font déambuler les paroissiens dans un espace correspondant pour bonne part au finage. C'est donc à une première approche du phénomène processionnel dans la perspective indiquée, comme contribution à la formation à long terme de l'espace communautaire paroissialisé, que nous voudrions consacrer ces quelques lignes.

La question du rapport entre procession et constitution des communautés n'est pas neuve, même si elle n'a jusqu'alors pas été véritablement creusée - à l'inverse du rapport entre pèlerinage et communitas, notamment à la suite des travaux de Victor et Edith Turner ${ }^{10}$. Fondés sur la catégorie ethnologique du « rite de passage », qui impose temporairement la sortie hors du groupe, la confrontation à l'altérité sur les marges puis le retour dans le groupe, ces tra-

D. Pichot dans le présent volume. Par ailleurs, les limites en question sont moins des limites tournées vers l'extérieur (pour exclure les autres) que vers l'intérieur (pour signaler les endroits où s'achève le ressort de l'église et empêcher les paroissiens d' « aller voir ailleurs »).

8. S. Leturcq, "Territoires agraires... », op. cit., insiste clairement sur les nombreux cas d'inadéquation morphologique de l'appartenance paroissiale et de l'appartenance communautaire au sens employé ici de communauté d'habitat et de production.

9. Cf. le cas de la Touraine étudié dans Des paroisses de Touraine aux communes d'Indre-et-Loire, la formation des territoires, É. Zadora-Rio dir., Tours, FERACF (Revue archéologique du Centre de la France, supplément 34), 2008. Il y apparaît cependant que même à la fin du XviII siècle, les paroisses sont loin d'avoir toujours des limites nettes et que la mise au net des limites communales a justement résulté d'un travail de systématisation administrative d'alors - fondé précisément sur une considération d'homogénéité et d'isotropie de l'espace, dont la conception ne peut pas être antérieure à Descartes.

10. V. Turner, Dramas, Fields and Metaphors, Londres/Ithaca (N.Y.), Cornell University Press, 1974, et V. et E. Turner, Image and Pilgrimage in Christian Culture, New York, Columbia University Press, 1978. 
vaux voient donc le pèlerinage comme un rite d'inversion de la structure principale, par lequel est réalisée l'intégration à et donc l'intégration de la communitas. Tout en reconnaissant des caractères communs entre pèlerinage et procession (déplacement, parfois jusqu'aux marges du finage), on a cependant récemment signalé l'impossibilité de repérer dans les processions la dimension d'inversion structurale en question, si bien que celles-ci seraient en fait bien plutôt une réalisation spatiale, et donc une démonstration par une communauté déjà constituée (et non pas à constituer), de structures sociales à la fois hiérarchiques et de coappartenance ${ }^{11}$. La contribution du phénomène processionnel à la constitution de la communitas serait donc nulle du point de vue anthropologique - ce qui nous incite d'autant plus à l'envisager, en l'occurrence du point de vue historique.

L'étude de cette contribution impose une double rupture par rapport à la tradition historiographique. En premier lieu, les processions doivent être conçues comme des phénomènes spécifiquement spatiaux (c'est-à-dire des pratiques spécifiques de l'espace) et non plus réduits à leurs seules formes rituelles (marcher ensemble, à telle date, dans tel ordre) ni à leurs fonctions conjuratoires ou propitiatoires. Les travaux sur les itinéraires processionnels menés en France ont ainsi initié un considérable renouvellement de l'approche des processions, en attirant notamment l'attention sur l'existence de choix spatiaux qui invitent à une lecture plus sociohistorique qu'une simple histoire des mentalités plus ou moins bien fondée ${ }^{12}$. En revanche, la compré-

11. J. Gengnagel, M. Horstmann, G. Schwedler, "Einleitung », dans Prozessionen, Wallfahrten, Aufmärsche. Bewegung zwischen Religion und Politik in Europa und Asien seit dem Mittelalter, J. Gengnagel, M. Horstmann, G. Schwedler dir., Cologne/Weimar/Vienne, Böhlau, 2008, p. 315, ici p. 12. C. Zika, « Hosts, Processions and Pilgrimages : Controlling the Sacred in FifteenthCentury Germany », Past and Present, 118 (1988), p. 25-64, estime qu'on assiste au Xve et au début du XVI $\mathrm{e}^{\mathrm{e}}$ siècle à une remise en question cléricale du pèlerinage, conçu comme un "voyage potentiellement incontrôlable au-delà des limites d'un territoire ", au profit de la seule procession en tant qu' « acte liturgique entièrement sous le contrôle des autorités locales » et que "célébration par la communauté de ses objets sacrés à travers un espace politique prescrit » (p. 63). On observera cependant que ce rapport établi entre pèlerinage et communauté (et par conséquent la négation de ce rapport quant à la procession...) repose sur l'assimilation implicite de la communitas et de la communauté locale - mais où est, à l'échelle de la chrétienté, le caractère d'inversion, même lorsqu'on est aux marges (Saint-Jacques, Rome, Jérusalem) ? Si le pèlerinage peut être intégrateur en convergeant ad limina sans pour autant sortir de l'espace commun, pourquoi alors ne pas considérer sous le même angle les processions qui conduisent les habitants aux marges du finage, comme celles des Rogations de l'époque moderne que présente S. Hindle, « Beating the Bounds... », op. cit. ?

12. M. Venard, "Itinéraires de processions dans la ville d'Avignon », Ethnologie française, 7 (1977), p. 56-62 ; N. Coulet, " Processions, espace urbain, communauté civique », dans Liturgie et musique (IX ${ }^{e}$-XIVe siècles), Toulouse, Privat (Cahiers de Fanjeaux, 17), 1982, p. 381-397; J. Chiffoleau, "Les processions parisiennes de 1412. Analyse d'un rituel flamboyant ", Revue Historique, 284 (1990), p. 37-76 ; J.M. Matz, « Le développement tardif d'une religion civique dans une ville épiscopale. Les processions à Angers (v. 1450-v. 1550) », dans La religion civique à 
hension des effets sociaux des pratiques spatiales reste insuffisante, notamment en ce qui concerne la genèse des communautés, parce que les travaux sur les itinéraires adoptent fondamentalement une conception de l'espace comme " écran de projection » ${ }^{13}$, que remettent précisément en cause les approches de la spatialité médiévale depuis la fin des années 1980.

Cet anhistorisme spatial nous conduit directement à la seconde rupture historiographique à opérer, qui consiste en un rejet de la posture « continuationniste », qui repose sur deux principes distincts (mais combinables) : il s'agit d'une part de la réduction de la procession (comme de tout rite) à des besoins anthropologiques constants, ici le besoin de sécurité, qui conduit à un fonctionnalisme anhistorique ${ }^{14}$. D'autre part, le "continuationnisme » consiste à voir dans des ressemblances formelles et institutionnelles entre des pratiques d'âges différents des signes de continuité et des continuités de sens, ce qui engendre dès lors une argumentation en termes d'héritage ou de réapparitions - alors que rien ne permet de postuler que le sens social d'une forme et donc les fonctions d'une pratique sont identiques.

Classiquement en effet, les processions ont été étudiées comme des rites de protection (liés à la figure circulaire du mouvement), à partir d'une appro-

l'époque médiévale et moderne (Chrétienté et Islam), A. Vauchez dir., Rome, École française de Rome, 1996, p. 351-366 ; P. Collomb, «Les processions des Rogations à Lyon au Moyen Âge: les parcours, le mythe et l'auctoritas cathédrale (XII ${ }^{\mathrm{e}} \mathrm{XVI}{ }^{\mathrm{e}}$ siècles) », Sources. Travaux historique, 51-52 (1997 = Processions et parcours en ville), p. 69-93; Fr. Bordes, " Une perception de l'espace urbain : cortèges officiels et processions générales à Toulouse du XIV au XVI siècle ", Mémoires de la Société d'Archéologie du Midi de la France, 64 (2004), p. 135-153. En Allemagne, le problème des itinéraires a beaucoup moins intéressé, ce qui se traduit par l'absence de tentatives de cartographie des processions lorsque, par extraordinaire, on se préoccupe d'en signaler le trajet ; ceci est certainement lié en partie au fait que les processions y ont très peu préoccupé les historiens (médiévistes), au contraire des chercheurs en « sciences religieuses » et des ethnographes. Les rares exceptions au désintérêt historien sont les travaux de N. Kyll, Pflichtprozessionen und Bannfahrten im westlichen Teil des alten Erzbistums Trier, Bonn, Röhrscheid, 1962 ; de D. Scheler (cf. infra) ; et de G. Signori, Maria zwischen Kathedrale, Kloster und Welt. Hagiographische und historiographische Annäherungen an den Typus der hochmittelalterlichen Wunderpredigt, Sigmaringen, Thorbecke, 1995, et «Ereignis und Erinnerung. Das Ritual in der städtischen Memorialkultur des ausgehenden Mittelalters (14. und 15. Jahrhundert) ", dans Prozessionen, Wallfahrten..., op. cit., p. 106-121.

13. Pour une critique de cette conception de l'espace-écran sur lequel se projetterait le social, voir G. Desmarais, "Projection ou émergence: la structuration géographique de l'établissement bororo ", Recherches sémiotiques/Semiotic Inquiry, 12 (1992), p. 189-215. Un exemple significatif de cette naturalité instinctivement prêtée à l'espace (en l'occurrence urbain) : D. Alibert, R. Benedetto, «Processions et parcours en ville: vers une construction du sacré », Sources. Travaux historique, 51-52 (1997 = Processions et parcours en ville), p. 3-4, justifient l'intérêt de la procession comme objet historique par le fait qu'elle «permet de saisir la rencontre de trois champs distincts : la société, le temps et la mémoire, le sacré ».

14. J. Chiffoleau, "Les processions parisiennes... », op. cit., p. 38. G. Signori, «Ereignis und Erinnerung... », op. cit., p. 107, ajoute à la panoplie du parfait « anhistorien » des processions la distinction privé/public. 
che abstraite et plus ou moins anthropologique ; ou à l'inverse, ce même type d'approche a également abouti à surévaluer l'autre côté de la médaille, à savoir le caractère propitiatoire des processions, lues par exemple comme des rites de fécondité ${ }^{15}$. Que la dimension propitiatoire ou protectrice éventuelle soit indéniable (puisqu'en cas de danger guerrier, épidémique, agricole, on organise des processions extraordinaires) ne devrait en aucun cas faire passer pour insignifiante la manière dont est organisée la procession.

La chose est sans doute encore plus marquée pour les processions régulières (calendaires), parce que la force de la structure temporelle (date ou rythme), voire certaines «traditions » (comme les hommes sauvages, géants ou dragons processionnels), focalisent l'attention et qu'on a tôt fait d'y repérer des héritages historiques (romains, celtes, indoeuropéens, etc.) ${ }^{16}$, et/ou d'en faire des manifestations des croyances et pratiques populaires, c'est-à-dire irrationnelles, superstitieuses, que l'Église n'aurait laissé subsister que récupérées et encadrées ${ }^{17}$. Mais on devrait aussi dire la même chose des études qui voient dans la procession une simple réitération d'un modèle biblique (les Hébreux portant l'arche d'alliance et traversant la Mer rouge, le Christ désigné comme « chemin », etc.), bref comme si le sens des processions était hérité et non pas produit à chaque fois (de même que la parole actualise la langue).

C'est donc tant au caractère spatial qu'historique du phénomène processionnel que nous nous attacherons ici. Ludolf Kuchenbuch commencera par montrer combien certaines processions rencontrées dans les documents des $\mathrm{X}^{\mathrm{e}}$-XII ${ }^{\mathrm{e}}$ siècle permettent d'avancer l'hypothèse d'une contribution peut-être non négligeable du phénomène processionnel à l'évolution qui mène du système domanial au système seigneurial encellulé, donc à l'espace communautaire. Puis Dieter Scheler examinera l'ensemble des processions organisées à

15. P. Saintyves, "Le tour et la ceinture de l'église », Revue archéologique, 1922, p. 79-113; J. Delumeau, Rassurer et protéger. Le sentiment de sécurité dans l'Occident d'autrefois, Paris, Fayard, 1989, p. 145-150 ; P.A. Sigal, L'homme et le miracle dans la France médiévale (XI'-XII siècles), Paris, Le Cerf, 1985, p. 155-163. A. Vauchez, "Liturgie et culture folklorique : les rogations dans la Légende dorée de Jacques de Voragine », dans Fiestas y liturgia / Fêtes et liturgie (Madrid, 1985), A. Esteban, J.-P. Étienvre dir., Madrid, Universidad Complutense/Casa de Velázquez, 1988, p. 21-34, rééd. dans A. Vauchez, Les laïcs au Moyen Âge, Paris, Le Cerf, 1987, p. 145-156.

16. Un exemple très significatif : Ph. Walter, « L'or, l'argent et le fer (étiologie d'une fête médiévale : les Rogations ", Le Moyen Âge, 99 (1993), p. 41-59 (le fait de parler des Rogations seulement en tant que fête signale d'ailleurs l'inintérêt pour le caractère spatial - ici processionnel -, effectivement absent de l'article).

17. Cf. les remarques de D. Scheler, «Inszenierte Wirklichkeit : Spätmittelalterliche Prozessionen zwischen Obrigkeit und Volk », dans Von Aufbruch und Utopie. Perspektiven einer neuen Gesellschaftsgeschichte des Mittelalters, für und mit Ferdinand Seibt aus Anlaß seines 65. Geburtstages, B. Lundt, H. Reimöller dir., Cologne/Weimar/Vienne, Böhlau, 1992, p. 119-129, ici p. 119-120. 
partir de Xanten et notamment leur contribution à la configuration somme toute tardive, à partir du XIII ${ }^{\mathrm{e}}$ siècle, d'espaces de domination spécifiques en fonction desquels peuvent s'affirmer des identités communautaires. Joseph Morsel envisagera enfin le problème de l'éventualité d'une contribution particulière des Rogations à la configuration ecclésiale de l'espace communautaire.

\section{Visite ou procession ? Remarques sur la genèse déambulatoire d'espaces de domination « patronale »}

Je vais traiter ici de certaines visitationes, qui vont nous introduire dans le vaste champ des traces difficiles à déchiffrer des pratiques de déplacement ou déambulation organisées en groupe entre le Ve et le XII siècle et à propos desquelles je poserai l'hypothèse qu'elles ont contribué à la structuration de l'espace domanial puis à sa restructuration dans le sens de sa " paroissialisation $»^{18}$. Il s'agit de témoignages courts, le plus souvent allusifs, à partir desquels on a jusqu'alors trop souvent et trop vite monté une image synthétique et censée avoir traversé les changements de cultures, époques et espaces.

\section{Le cas d'Echternach dans l'optique courante}

Le premier témoignage nous conduit à l'abbaye d'Echternach (au nord de Trèves) vers 1100. Je cite : "le mardi de la Pentecôte, ils visiteront religieusement leur saint patron dans l'église de... » - suivi de 82 toponymes, souvent complétés par la mention d'autres capellæ et par les pains et deniers apportés par celles-ci ${ }^{19}$. Nikolaus Kyll, qui a exhumé ce document, estime que la localisation des toponymes à proximité immédiate de l'abbaye ne laisse aucun doute sur le fait qu'il s'agit d' « une liste de lieux soumis à un devoir de procession ", des registres plus tardifs de l'abbaye (XVe-XVIII siècles) témoignant de la longue durée de cette pratique processionnelle. Une information fournie peu après 1100 par Thiodfrid d'Echternach dans sa vita en prose de saint Willibrord, le fondateur d'Echternach, permet de préciser le témoignage. Dans le chapitre 24, il rapporte qu'au moment de la Pentecôte, un rassemblement de clercs et de gens innombrables provenant des environs et de la région de

18. Cette notion à laquelle nous recourons ici encore ne devrait pas être considérée comme la proposition déguisée d'un nouveau modèle (inparochiamento) : étant donné ce que nous avons dit de la paroisse en tant que « modèle réduit » et représentation locale de l'ecclesia, il y a coïncidence entre inparochiamento et l'inecclesiamento proposé par M. Lauwers (à condition de ne pas restreindre l'ecclesia en question au seul bâtiment, par analogie avec le castrum à l'arrièreplan de l'incastellamento).

19. Feria tertia in pentecostem visitabunt religiose patronum suum isti/illi ecclesiæ de [...] : N. Kyll, Pflichtprozessionen..., op. cit., p. 54-55. 
l'abbaye se serait rendu en toute humilité auprès du saint, selon un rite ancien et inviolable, avec des dons et avec des litaniæ (donc soit " avec des chants » soit « en procession ») $)^{20}$.

Les points cruciaux dans ces témoignages d'Echternach sont la focalisation spatiale et temporelle de la visitatio sur le principal patron d'un monastère (i.e. sur ses reliques), la participation de clercs, la désignation des participants laïques par rapport à des églises locales (dont ils dépendaient probablement), le déplacement sous forme processionnelle avec des chants et prières, l'apport de pains ou deniers dont la signification liturgique est signalée par le mot oblatio. À partir de chartes, censiers, listes et pouillés dans la plupart des cas postérieurs, N. Kyll a repéré de semblables visitationes dans les autres anciennes abbayes et églises du diocèse de Trèves, comme Prüm, Mettlach, SaintMaximin, Taben et Luxembourg 21.

La contribution apportée par N. Kyll en 1964 à l'histoire locale et régionale est certes admirable, mais il a donné de ces visitationes une interprétation qui a été longtemps admise bien qu'elle soit à mon sens critiquable. Il y voit notamment des processions obligatoires (Pflichtprozessionen) des dépendants envers leur seigneur foncier, envers lequel « ils témoignent à cette occasion, par une oblatio, de leur rapport de dépendance et de leur devoir de fidélité ${ }^{22}$. Ces processions seraient, par rapport aux visitationes religiosæ gallo-romaines du clergé et de l'aristocratie auprès des évêques attestées depuis le ve siècle, le résultat d'un « détournement » de la domination épiscopale au profit de celle de l'abbaye.

Ce glissement «seigneurial» se manifesterait dans trois phénomènes : 1) la fixation des dates de visitatio aux jours de fête du saint patron principal de chacune des églises concernées ; 2) la transformation de l'oblatio volontaire en une redevance obligatoire analogue au census seigneurial ; 3) l'existence

20. Ibid., p. 52 ; le passage complet est : Indicio quoque usque in presens est adventu paraclyti Spiritus celeberrima pentecostes ebdomada, in qua non modo ex tota circumquaque vicinia, sed ab universa Gallię ac Germanię provincia ritu perpetuo et quasi lege insolubili et inviolabili a progenie in progeniem transmissa et ceu iuramento Domini confirmata et tradita, innumerabilis cleri et populi conventus et frequentia cum oblationibus et lętanïs summa cum devotione sancta eius terit limina, ob divulgata et enarrata profecto a patribus filiis mirabilia, quę ibi in tantę solempnitatis lęticia, omni revoluta vertentis anni orbita, ostensa sunt ante altare consecratum in septemplicis Pneumatis honore et gloria (Acta Sanctorum Novembris, t. 3, Bruxelles, 1910, p. 475).

21. N. Kyll, Pflichtprozessionen..., op. cit., p. 23-80 ; pour compléter : A. Heinz, « Die von Erzbischof Egbert gestiftete Bannfeier (statio bannita). Ursprung und Ende eines trierischen Prozessionsbrauches », dans Egbert Erzbischof von Trier 977-993. Gedenkschrift der Diözese Trier zum 1000. Todestag, F.J. Ronig et alii dir., Trèves, Selbstverlag des Rheinischen Landesmuseums Trier, 1993, p. 67-80.

22. N. Kyll, Pflichtprozessionen..., op. cit., p. 50. 
d'une aire de ponction sur les églises dépendantes, constituée à partir de la «zone d'influence» de l'abbaye au moment de sa fondation pour donner «l'espace processionnel » ultérieur. Ceci se serait produit en étroite relation avec la transformation par subdivision et comblement des anciennes macroparoisses, aboutissant au réseau paroissial à mailles serrées du Moyen Âge central. L'ensemble du processus, bien qu'initié par les fondations d'abbayes et leurs dotations foncières, aurait débuté à la fin de l'époque carolingienne et, quoique largement accompli au XII siècle, aurait souvent duré jusqu'au XVIII siècle.

\section{Changement d'échelle, changement de sens}

Dans l'optique d'une relecture de ces résultats, je voudrais partir d'un autre témoignage écrit, datant du XII siècle, que N. Kyll, qui se limitait au diocèse de Trèves, n'a pas utilisé. Il provient en effet d'un codex compilé à et pour l'abbaye de Deutz (en face de Cologne, sur la rive orientale du Rhin) dans les années 1146-1169 et qui, outre la notice qu'on va évoquer, renferme divers textes dont un inventaire détaillé des biens et cens de l'abbaye. Dans ce codex est signalée la chose suivante :

Hee sunt parrochie que beato Heriberti collectas suas vel elemosinas annuatim pro conservatione frugum deferre solent, ut eius interventu defendat deus segetes eorum ab omni tonitruo, fulgere, grandine et tempestate. ${ }^{23}$

La phrase citée est suivie d'une liste de 27 parrochiæ, dont deux couvrent plusieurs agglomérations (adiacentes villæ). La notice en question s'achève par la remarque :

Isti omnes elemosinis suis beatum Heribertum singulis annis antiquitus visitabunt, sed nunc pluribus se subtrahentibus pauci adhuc in ea devotione perseverant. ${ }^{24}$

23. «Telles sont les paroisses qui doivent chaque année apporter à saint Héribert ses collectes ou aumônes pour la conservation des récoltes, afin que par son intervention Dieu protège leurs semences de tout tonnerre, foudre, grêle et tempête ": J.T. Lacomblet, " Die BenediktinerAbtei zu Deutz. Ihre Stiftung und ersten Wohltäter, ihre Äbte, Besitzungen und Reliquien », Archiv für die Geschichte des Niederrheins, 5 (1866), p. 265-322, ici 289-290. Sur l'histoire de l'abbaye, voir J. Milz, « Deutz », Germania Benedictina, 8 (1980), p. 293-313; sur la tradition documentaire, voir M. Sinderhauf, Die Abtei Deutz und ihre innere Erneuerung: Klostergeschichte im Spiegel des verschollenen Codex Theodorici, Vierow bei Greifswald, SH-Verlag, 1996 ; sur la seigneurie de l'abbaye, voir J. Milz, Studien zur mittelalterlichen Wirtschafts- und Verfassungsgeschichte der Abtei Deutz, Cologne, H. Wamper, 1970.

24. «Toutes visitaient chaque année et de toute antiquité saint Héribert avec leurs aumônes, mais comme beaucoup s'y sont soustraites, peu ont jusqu'à présent persévéré dans cette dévotion » : J.T. Lacomblet, « Die Benediktiner-Abtei zu Deutz... », op. cit., p. 290. 
Ce témoignage date d'environ un demi-siècle après celui d'Echternach. Cinq points ressortent de la comparaison des deux : en premier lieu, en dépit de la dévotion évoquée à l'égard du patron du monastère saint Héribert, il ne s'agit pas dans ce second cas d'une reconnaissance de la domination du saint patron, mais de l'obtention de son intercession auprès de dieu pour que les récoltes soient préservées des intempéries; bien que rien ne soit dit ici du terme de la visitatio, le contenu de la prière laisse penser qu'il s'agissait des mois entre Pâques et la saint-Jean, période de la croissance et du mûrissement des récoltes. En second lieu, ce sont des «paroisses » qui s'engagent dans l'affaire, et non pas des "églises ». Troisièmement, dans l'espoir de l'effet protecteur, ces participants paroissiaux versaient à l'intercesseur des " aumônes » et " collectes », ils ne sont pas contraints à des oblations. Quatrième point : il s'agit certes d'une ancienne dévotion, mais elle a clairement décliné face à un refus de l'accomplir, dont les causes sont inconnues. Enfin, les paroisses qui entreprennent cette procession propitiatoire ne coïncident pas avec les possessions (curtes) qui sont inventoriées dans le censier contemporain.

Comment comprendre ces changements dans le lexique, dans la fonction et dans la mise en œuvre pratique ? On peut commencer par les replacer, prospectivement, dans le contexte de ce qui advient, c'est-à-dire dans l'hypothèse d'un "encellulement » général - qui se combine sans difficulté avec le passage terminologique de l'ecclesia ou capella à la parochia ou aux parochiani. Le nombre croissant depuis la fin du $\mathrm{X}^{\mathrm{e}}$ siècle des mentions isolées de processions peut aussi être considéré comme un autre indice-clé de la «paroissialisation ».

Par ailleurs, il est dit dans une notice de 1128 concernant une procession organisée en direction de l'église de Luxembourg: « Les paroissiens (parochiani) des villages (villæ) s'assemblent avec les reliques et les dons qu'ils ont à présenter ce jour-là ${ }^{25}$. On mène donc les reliques de l'église paroissiale jusqu'au patron principal. On sait en outre qu'une croix ouvre le cortège, que mène le doyen local et qu'identifie une bannière que l'on "prête » au saint patron du monastère et que celui-ci restitue après l'oblation. Le groupe en déplacement « figure » les inhabitantes de l'agglomération, il doit se composer d'hommes qui représentent chacun leur exploitation (mansus, curtilis, domus) et qui prient, implorent et chantent en langue vernaculaire. Les cortèges des paroisses les plus éloignées se rencontrent à mi-chemin de l'église principale et continuent ensuite leur chemin ensemble.

Ceci engendre un trajet radioconcentrique - le saint patron, la relique centrale, fonctionnant comme un aimant qui intègre les paroisses à l'espace de salut du saint patron par le biais de tronçons définis et fixes des chemins 
locaux. Par la combinaison de déplacements linéaires et circulaires qui semblent analogues à ceux observés dans les Rogations, s'accomplit aussi une démarcation par rapport à l'extérieur tandis qu'est définie la centralité secondaire des paroisses. Toutes choses qui convergent avec des phénomènes bien connus : microtoponymie, codifications et chartes de franchises, délimitations de paroisses dans des chartes, etc.

\section{Noyaux patronaux sans cellules}

Voyons maintenant comment articuler les changements observés avec ce qui les précède, en considérant la période du VIII jusqu'au début du XI ${ }^{\mathrm{e}}$ siècle. On convoquera à cet égard un dernier témoignage, du plus grand intérêt. Il nous conduit à l'époque de la fondation de l'abbaye de Schildesche (près de Bielefeld) peu avant le milieu du IX ${ }^{\mathrm{e}}$ siècle ${ }^{26}$. La vita consacrée à la fondatrice et première abbesse, Marcswidis, rappelle la difficile quête des reliques de saint Jean désirées pour la nouvelle fondation. Après qu'enfin elles furent parvenues à Schildesche, en passant par le siège épiscopal de Paderborn, elles furent présentées officiellement par la matrona Marcswidis dans la curtis voisine de Sellhausen, où les gens des paroisses voisines se déplacèrent en masse, les honorèrent, les gratifièrent de dons, les veillèrent la nuit, les illuminèrent, les chantèrent et s'enivrèrent de leur odeur. Le matin suivant, la matrona adressa les paroles suivantes au populus assemblé :

[...] statuimus, ut annuatim secunda feria pentecostes, Spiritu sancto cooperante, eundem patronum parochiis vestris longo ambitu circumferentes et domos vestras lustrantes pro gentilico ambarvali, in lacrimis et varia devotione vos ipsos mactetis et ad refectionem pauperum elemosynam comportetis et in hac curti pernoctantes super reliquias vigiliis et canticis spiritualibus solennisetis, ut predicto mane determinatum a vobis ambitum pia lustratione complentes, ad monasterium cum honore debito reportetis. Confido autem de patroni huius misericordia, quod sic ab eo girate terre semina uberius provenient, et varie aeris inclementie cessent. ${ }^{27}$

26. H. Redecker, Das Stift Schildesche im Mittelalter, Emsdetten, Lechte, 1936 ; Stift und Kirche Schildesche 939-1810. Festschrift zur 1050-Jahr-Feier, U. Andermann dir., Bielefeld, Bielefeld/Herford, Welscher/Busse \& Seewald, 1989.

27. Fundatio Monasterii Schildecensis, éd. O. Holder-Egger dans Monumenta Germaniæ Historica, Scriptores, t. XV, 2, Hanovre, 1888, p. 1045-1052, ici 1050-1051 : « [...] nous prescrivons que chaque année, le deuxième jour de la Pentecôte, avec l'assistance du saint Esprit vous transportiez le saint patron [i.e. saint Jean] sur un long trajet dans vos paroisses à la place de la déambulation coutumière, et que vous nettoyiez vos maisons, vous vous purifiez vous-mêmes par des larmes et toutes formes d'humilité, apportiez des aumônes pour la nourriture des pauvres et passiez la nuit sur cette cour [i.e. Sellhausen], veilliez alors sur les reliques avec des chants sacrés solennels, afin que le jour suivant, tôt le matin, vous acheviez par une pieuse déambulation le trajet que vous aurez décidé et les rapportiez au monastère avec tout le respect qui leur 
La suite du récit montre que l'abbesse institue ici une déambulation locale, centrée sur le domaine, circulaire et définie par les gens eux-mêmes avec les reliques du patron de monastère qui leur ont été « prêtées » - «prêt » qu'on doit comprendre comme la contrepartie de l'accueil plein de piété des reliques par les paroissiens des environs du monastère. Cette déambulation ${ }^{28}$ fournit à notre analyse rétrospective les points d'appui suivants : 1) la proximité temporelle des Rogations (les trois jours précédant le jeudi de l'Ascension) ainsi que de leur caractère pénitentiel est frappante. 2) Il semble clair aussi que pour les paroissiens, " leurs » saints patrons locaux et leurs reliques ne jouent aucun rôle : c'est bien plutôt le saint patron central qui est institué comme intercesseur hégémonique. 3) Les paroissiens disposent encore d'une marge de manœuvre pour les délimitations locales, encore inexistantes.

On peut rapprocher ceci des données fournies par la documentation liturgique générale: d'une part, dans le pontifical romano-germanique - cette grande compilation de base d'ordines non eucharistiques et de mesures pratiques épiscopales rédigée entre 950 et 962 dans la région de Mayence et au rayonnement si important -, les indications détaillées sur la litanie majeure (procession de saint Marc) et sur les litanies mineures (les Rogations) ne présentent aucune référence à l'espace ${ }^{29}$. D'autre part, la documentation synoda-

est dû. J'ai foi en la miséricorde de ce patron qu'en vertu de cette circumambulation de la terre, les grains surgissent plus nombreux et les diverses intempéries soient écartées ».

28. Je ne m'étends pas ici sur les - indéracinables - filiations établies étroitement entre ces déambulations et de prétendus usages païens : cf. K. Ranke, "Germanische Flurumgänge ", dans Untersuchungen zur eisenzeitlichen und frühmittelalterlichen Flur, H. Beck (dir.), t. 2, Göttingen, Vandenhoeck \& Ruprecht, 1980, p. 361-369 ; C. Daxelmüller, "Flurumgang », Reallexikon der germanischen Altertumskunde, $2^{\mathrm{e}}$ éd., t. 9, Berlin, De Gruyter, 1995, p. 261-263 ; R. Schmidt, "Umfahrt », Handwörterbuch zur deutschen Rechtsgeschichte, t. 5, Berlin, E. Schmidt, 1998, col. 421-426.

29. Les ordines romani du haut Moyen Âge, V : Les textes. L'ordo L, éd. M. Andrieu, Louvain, Spicilegium sacrum Lovaniense, 1961, p. 315-342, et Le Pontifical romano-germanique du dixième siècle, t. 2, éd. C. Vogel, R. Elze, Le Vatican, Biblioteca Apostolica Vaticana, 1963, p. 119-131. Seuls quelques antiphones comprennent de timides références «spatiales » : le lundi, au moment de l'élévation des reliques, l'antiphone Surgite, sancti, de mansionibus vestris, loca sanctificate, plebem benedicite et nos homines peccatores in pace custodite, alleluia. De Hierusalem exeunt reliquiæ et salvatio de monte Syon..., plus tard encore l'antiphone Sanctos portamus, sanctorum laudes dicimus et nos in terra psallimus... ; l'antiphone Surgite, sancti... est répété le mardi et le mercredi, suivi ce dernier jour de l'antiphone Domine, Deus omnipotens patrum nostrorum, respice de celo et de sede sancta tua pro pace regum et quiete populorum, pro loco nostro et habitantibus in eo (Les ordines romani..., op. cit., p. 317, 331, 333, 338 ; Le Pontifical..., op. cit., p. 120, 126, 127, 129). Le Pontifical..., p. 367, fournit également la formule de Benedictio in campo sive in agro quando agitur letania, qui montre donc l'existence au moins théorique de processions dans les champs au $\mathrm{x}^{\mathrm{e}}$ siècle le jour d'une litanie - qu'elle fût majeure (le même pontifical déclare en effet à propos de celle-ci que ex qualitate temporis colligimus eam necessariam esse ad fruges conservandas : Les ordines romani..., p. 315 , Le Pontifical..., p. 119) ou mineure. 
le et canonique que le manuel de visite pastorale composé en 906 par Réginon de Prüm pour le diocèse de Trèves mobilise sur ces deux litanies n'offre rien de rien de précis sur le rapport entre procession et organisation paroissiale, bien que N. Kyll montre que ces processions aient été " habituelles » pour le clergé et le peuple dans le diocèse de Trèves ${ }^{30}$.

Enfin, il convient de prendre en compte dans notre approche un immense champ d'iter agere ${ }^{31}$, à savoir toutes les corvées en travail sur les domaines ainsi que les multiples corvées de charroi (carropera et angariæ) ou de transport à cheval et les services de messagerie (scaræ) ${ }^{32}$. Si l'on met ces modes de déplacement des gens en rapport avec les autres, on peut alors repérer une configuration spatiale dominante qui me semble être l'existence d'une polarité dominante des centres épiscopaux ou monastiques par rapport à leurs marges domaniales - une polarité par rapport à laquelle les églises rurales ne sont que des dépendances des domaines (comme les moulins), et non pas des centres secondaires.

Quant aux gens des domaines, les «familiers » de leurs maîtres (domini, seniores), ils circulaient sur les domaines en tant qu'espaces de service et accomplissaient des trajets de service vers le centre en fonction de schémas temporels et de circulation spécifiques33. Les visitationes proprement dites vers le centre étaient assurées par les ministri domaniaux à Noël et Pâques. Inversement, les seigneurs effectuaient eux-mêmes une visite (adventus) sur leurs terres, pour la collecte des rentes et la tenue des plaids laïques et synodaux ${ }^{34}$.

30. Das Sendhandbuch des Regino von Prüm, éd. W. Hartmann, Darmstadt, Wissenschaftliche Buchgesellschaft, 2004, p. 146-148 ; N. Kyll, Pflichtprozessionen..., op. cit., p. 81-101.

31. Pour une mise en perspective générale - et par conséquent anhistorique - du rapport métaphorique et normatif entre la destinée chrétienne et le chemin et le cheminement, voir L. et R. Kriss-Rettenbeck, I. Illich, "Homo viator. Ideen und Wirklichkeiten ", dans Wallfahrt kennt keine Grenzen, L. Kriss-Rettenbeck, G. Möhler dir., Munich/Zurich, Schnell \& Steiner, 1984, p. 10-22. Sur le caractère central de l'iter au sein des représentations chrétiennes de l'espace, voir aussi A. Guerreau, « Le champ sémantique de l'espace dans la Vita de saint Maieul (Cluny, début du XI ${ }^{\mathrm{e}}$ siècle) », Journal des Savants, juillet-décembre 1997, p. 363-419.

32. L. Kuchenbuch, Bäuerliche Gesellschaft und Klosterherrschaft im 9. Jahrhundert. Studien zur Sozialstruktur der Familia der Abtei Prüm, Wiesbaden, Steiner, 1978, p. 124-145 ; J.-P. Devroey, Études sur le grand domaine carolingien, Aldershot, Variorum, 1993, ${ }^{\circ} \mathrm{X}$-XI, et Puissants et misérables. Système social et monde paysan dans l'Europe des Francs ( $\mathrm{II}^{e}-\mathrm{IX}{ }^{e}$ siècles), Bruxelles, Académie Royale de Belgique, 2006, p. 547-570.

33. J. Demade, « Les „corvées“ en Haute-Allemagne... », op. cit., p. 344-345.

34. L. Kuchenbuch, «Porcus donativus: Language Use and Gifting in Seignorial Records between the Eighth and the Twelfth Centuries ", dans Negotiating the Gift. Pre-Modern Figurations of Exchange, G. Agazi, V. Groebner, B. Jussen dir., Göttingen, Vandenhoeck \& Ruprecht, 2003, p. 193-246, ici p. 209-228. 


\section{Prélude à la cospatialisation communautaire}

L'obligation faite aux gens des environs, qu'ils soient «familiers » domaniaux ou fidèles des églises locales, de la visitatio du centre - organisée ou non sous forme de, réduite à ou complétée par une procession - entre le VIII et le $\mathrm{XI}^{\mathrm{e}}$ siècle me semble correspondre à l'évolution spécifique des rapports de production dans les campagnes, aboutissant à une plus grande cohérence entre centre, environs immédiats et périphéries domaniales, à quoi contribue ici la mobilisation par les pouvoirs domaniaux de leur instance la plus universelle, le saint patron principal (et ses reliques).

Rappelons que dans le cadre domanial, le système des corvées faisait de la réserve le pôle et de la familia le cadre d'organisation du travail. Ce n'est plus le cas dans le système seigneurial: désormais, le pôle d'organisation est l'exploitation agricole elle-même et son cadre est la communauté d'habitants 35 . Mais le caractère communautaire de l'organisation productive se réalise à travers la structuration de l'espace productif, qui se signale à travers la pulvérisation des parcelles et la dispersion des parcelles de chaque exploitant à travers tout le finage, donc à une imbrication étroite des parcelles de chacun. Cela signifie pour l'agriculteur qu'il exploite désormais non un manse compact mais plusieurs parcelles dispersées dans le finage : au lieu de se déplacer de son manse à la réserve comme à l'époque carolingienne, réalisant ainsi spatialement sa dépendance vis-à-vis du maître, comme l'avait montré Julien Demade, il arpente désormais l'ensemble du finage, signalant ainsi son interdépendance vis-à-vis des autres villageois.

On observe alors sans difficulté combien les déambulations de la période domaniale venaient d'une part redoubler la focalisation des tenanciers sur le centre domanial (domination domaniale), d'autre part combien l'inclusion ensuite des « autres » fidèles par le biais d'une vénération élargie des reliques (impliquant alors aussi la substitution d'aumônes volontaires à des oblations obligées) permettait de dépasser le simple cercle des « familiers », préludant ainsi à une structuration en cellules plus larges que les simples villæ. On pourrait qualifier cette forme intermédiaire entre domination domaniale et domination seigneuriale (dans le cadre des communautés d'habitants) de

35. J. Demade, "Les „corvées“ en Haute-Allemagne... », op. cit., p. 352-353 ; J. Morsel, L'aristocratie médiévale. La domination sociale en Occident ( $V^{e}-X V^{e}$ siècle), Paris, Colin, 2004, p. 188193 ; L. Kuchenbuch, J. Morsel, "Ländliche Räume », dans Enzyklopädie des Mittelalters, G. Melville, M. Staub dir., t. 2, Darmstadt, Wissenschaftliche Buchgesellschaft, 2008, p. 249256, ici 254-255. 
« domination patronale », qu'Uta Kleine a nommée, dans le cadre de ses recherches sur les miracles, Patrozinium (« patronage ») ${ }^{36}$.

Avec cette forme de dépassement de la porosité spatiale du dominium et du senioratus ainsi que d'encadrement des familiers " propres » et des fidèles voisins dans un espace symbolique, celui du saint patron, on a donc affaire à un facteur important d'évolution de la domination sociale avant le processus de densification locale autour des églises rurales, marchés, châteaux, etc. - mais que $\mathrm{N}$. Kyll interdit de percevoir avec sa thèse de la récupération seigneuriale de processions anciennes qui néglige le changement de nature spatiale. Cette forme d'élargissement du cercle des hommes soumis à l'obligation de procession n'a peut-être pas été systématique et a en tout cas été transitoire, comme le montre non seulement le cas de Deutz (dont on pourrait considérer la mention de l'abandon de l'ancienne procession comme le résultat de la paroissialisation de son organisation), mais aussi celui de Prüm, Mettlach, SaintMaximin.

Tout ceci n'est cependant qu'une hypothèse, développée à partir du beau travail préparatoire de N. Kyll. Ceci renvoie aussi aux remarques liminaires : toute affirmation explicite ou considération implicite de l'existence d'une continuité à propos de processions doit être mise en doute, de même que doit être remise en cause la démarche traditionnelle qui consiste à doter les déambulations de fonctions fixes - tout ceci au mépris de la nature spatiale du phénomène processionnel. Mais cela implique aussi de travailler de manière plus poussée sur les éventuels facteurs qui pouvaient assurer, aux siècles du haut Moyen Âge non « spatialisés »37 et non « encellulés », la cohérence domaniale et la cohésion des hommes - facteurs qui restent à découvrir.

36. U. Kleine, Gesta, Fama, Scripta. Rheinische Mirakel des Hochmittelalters zwischen Geschichtsdeutung, Erzählung und sozialer Praxis, Stuttgart, Steiner, 2007, p. 97-102, 173-177, 242-253, 348354 ; Id., "Patronus oder Patriota ? Rheinische Wundersammlungen und ländliches Raumgefüge im Rheinland (11.-13. Jahrhundert) », dans Patriotische Heilige. Beiträge zur Konstruktion religiöser und politischer Identitäten in der Vormoderne, D.R. Bauer, K. Herbers, G. Signori dir., Stuttgart, Steiner, 2007, p. 87-111.

37. Cet adjectif ne vise pas à suggérer que la société du haut Moyen Âge n'était pas spatialisée (ce qui serait une absurdité), mais que la référence à l'espace n'était pas une constituante clé du système des représentations sociales - au contraire de ce que l'on observe à partir du XI ${ }^{\mathrm{e}}$ siècle, dans le cadre de ce que divers médiévistes qualifient désormais de « spatialisation du social ». 


\section{La production processionnelle d'espaces de domination : le cas de Xanten}

Xanten n'est pas un cas inintéressant dans la perspective ici envisagée. Collégiale fondée à partir de possessions de l'archevêque de Cologne, elle ne constituait pas seulement le centre d'une agglomération (érigée en ville en 1228) mais était également le siège d'un archidiaconé - l'archidiacre étant toujours le prévôt de Xanten. L'archidiaconé était une vaste circonscription au nord-ouest du diocèse, comprenant cinq doyennés et qui, à la fin du Moyen Âge, coïncidait largement avec le duché de Clèves, lequel s'était pour une bonne part constitué aux XII et XIII ${ }^{\mathrm{e}}$ siècles à partir de l'avouerie sur les possessions archiépiscopales et collégiales dans cette région. Parallèlement, les possessions archiépiscopales s'étaient notablement réduites au Moyen Âge central, à l'inverse de celles de la collégiale, qui s'étaient étendues sur les terres et gens et surtout les églises paroissiales. Conséquemment, la distribution spatiale des possessions de la collégiale coïncidait elle aussi largement avec le ressort de l'archidiaconé.

La collégiale articulait ainsi plusieurs formes sociales de rapport à l'espace. $\mathrm{Au}$ centre se trouvait le secteur immunitaire de la collégiale, autour duquel s'étendait l'agglomération, la ville de Xanten. Comme l'église de la collégiale était aussi église paroissiale, elle relevait du doyenné rural de Xanten, qui était de son côté un élément de l'archidiaconé. Du point de vue temporel, la collégiale détenait la justice à l'intérieur des murs de l'immunité et sur ses cours - mais une justice restreinte à la basse justice. La haute justice, elle, était rendue par l'avoué, le comte puis duc de Clèves. La collégiale représentait donc un pouvoir judiciaire d'une certaine manière concurrent des tribunaux de l'avoué, de l'agglomération, des échevins urbains, d'une part, et des tribunaux territoriaux de Clèves et du tribunal de cour d'autre part. La collégiale exerçait elle-même son pouvoir seigneurial dans l'espace en question, mais elle était en même temps soumise à ou en concurrence avec d'autres pouvoirs seigneuriaux dans cet espace ${ }^{3}$.

\section{Le système processionnel de Xanten}

C'est au sein de cette situation spatiale que prend son sens la pratique processionnelle de la collégiale, qu'on appréhende pour la première fois à travers le Liber Ordinarius, le manuel liturgique de la collégiale, dans le dernier tiers 
$\mathrm{du} \mathrm{XIII}^{\mathrm{e}}$ siècle 39 . Formellement, la pratique xantenoise ne se distingue qu'assez peu de celle qu'on observe non seulement dans les églises voisines de la région mais également dans toute la chrétienté latine. Le dimanche précédant le premier dimanche de l'Avent marque le début de la série des processions, qui toutefois restent limitées au seul secteur immunitaire jusqu'au dimanche des Rameaux, qu'il s'agisse de déambulations dans le cloître ou dans tout le secteur intérieur à l'immunité - et ce avec une solennité variable, comme le montre par exemple la tenue vestimentaire prescrite selon les cas aux clercs. Cette restriction spatiale vaut aussi pour les processions de la Purification (Chandeleur), du mercredi des Cendres et des processions du vendredi durant le Carême. En revanche, la procession des Rameaux tourne autour de la ville, ou alors va du porche de l'église à la chapelle Saint-André, toujours en ville, où a lieu la première station, pour ensuite passer par le marché et arriver à la porte Saint-Michel de l'immunité, lieu de la deuxième station, et enfin finir à l'église. Les processions dominicales de Pâques à l'Ascension ne se déroulent de nouveau qu'au sein de l'immunité.

La procession solennelle de l'Ascension faisait de nouveau tourner la châsse de saint Victor et les autres reliques autour de la ville. Il en allait de même pour les processions de la Pentecôte. À l'inverse, les processions du dimanche après l'Ascension et dans l'octave de Pentecôte n'avaient lieu que dans l'immunité. La dernière grande série de processions de l'année ecclésiastique était formée à Xanten par les processions du vendredi en été : de la Saint-Jean (24 juin) jusqu'à la Saint-Rémy ( $1^{\mathrm{er}}$ octobre), elles se rendaient chaque semaine à la chapelle Saint-André ou alors à Saint-Géréon, la seconde chapelle de la ville. Seule la première et la dernière de ces processions estivales tournaient autour de la ville.

Les litanies revêtaient inversement un caractère très particulier du point de vue de l'espace parcouru. Le jour de la litanie majeure, dite aussi Romana dans la chrétienté latine, c'est-à-dire à la Saint-Marc (25 avril), on se rendait avec la châsse de saint Victor et les autres reliques jusqu'à Lüttingen, une petite agglomération au nord de Xanten, dotée d'une chapelle ; mais on n'y allait que les jours non chômés : si la Saint-Marc tombait un dimanche ou le jour de Pâques, alors la procession tournait autour de la ville ${ }^{40}$.

Quant aux Rogations (la litanie mineure ou Gallicana de la chrétienté latine), qui constituaient le point culminant et des plus soigneusement réglés des processions, elles étaient accomplies de manière tout aussi solennelle que 
celle de la Saint-Marc. Le lundi avant l'Ascension, on allait à Lüttingen ou à Wardt, une petite agglomération près de Lüttingen également pourvue d'une chapelle ; le mardi, on allait à Fürstenberg, un petit monastère de cisterciennes fondé au XII siècle en tant que prieuré bénédictin et situé au sud de la ville ; le mercredi, enfin, on se rendait également à un petit monastère de bénédictines tout autant fondé au XII siècle, Hagenbusch, à l'ouest de la ville.

À première vue, les processions de Xanten ne paraissent pas présenter de traits spécifiques par rapport à celles que l'on connaît pour d'autres endroits. On sait d'ailleurs peu de choses précises sur les participants aux processions " publiques » (i.e. à l'extérieur de l'immunité), notamment sur la participation de la commune urbaine. Mais dans la mesure où, lors des défilés solennels, non seulement on transportait la châsse de saint Victor ${ }^{41}$ mais également on arborait des bannières, étant donné aussi que les sonneurs de cloche de la collégiale y prenaient part avec leurs masses et que la collégiale rétribuait des souffleurs de trompette pour qu'ils jouent durant la procession ${ }^{42}$, il s'agissait donc d'un rituel doté de publicité, suggérant une participation non limitée aux seuls clercs.

Du point de vue spatial, l'alternance des processions entre le secteur ecclésial restreint et un cadre plus étendu est tout aussi courante que le choix, pour les stations, d'églises en ville (les chapelles Saint-André et Saint-Géréon) ou dans les environs immédiats (les monastères de Fürstenberg et de Hagenbusch). De même, les processions autour des villes sont courantes et ont déjà fait l'objet de maintes analyses et interprétations historiennes.

\section{L'ambivalence du sens spatial des litanies à Xanten}

La seule chose étonnante est le choix des chapelles de Lüttingen et de Wardt, hors de la zone urbaine. À première vue, les processions au départ de la collégiale démontreraient simplement la subordination de ces chapelles, incorporées à la collégiale comme Saint-André et Saint-Géréon et soumises à sa justice spirituelle. Toutefois, les processions des Rogations auraient alors dû, s'il s'était agi d'une symbolisation du ressort paroissial de l'église de Xanten, également inclure trois autres agglomérations: Sonsbeck, Labbeck et Hurde ${ }^{43}$. Le mobile du choix de lieu ne peut donc pas se réduire à manifester le rapport de dépendance des églises vis-à-vis de l'église principale.

41. Die Stiftskirche des Hl. Viktor zu Xanten: Die Bursenrechnungen des St. Viktor-Stiftes zu Xanten 1401/o2 bis 1455/56, éd. D. Lück, Kevelaer, Butzon \& Bercker, 1993, col. 46.

42. Quellen zur Rechts- und Wirtschaftsgeschichte des Archidiakonats und Stifts Xanten, éd. C. Wilkes, Bonn, 1937, p. 230, 250, 451.

43. Ibid., p. 84. 
Le sens des choses ne devient perceptible que lorsque l'on prend en compte les rapports seigneuriaux temporels. Car encore en 1319, le comte de Clèves percevait, lors de la tenue de son tribunal en tant qu'avoué, à Xanten (en l'occurrence des mains du représentant local de l'archevêque de Cologne, seigneur de la ville jusqu'en 1444) les deux tiers des redevances qui lui étaient dues à ce titre, et le dernier tiers à Lüttingen (en l'occurrence sur des biens d'origine également colonaise mais en possession de la collégiale de Xanten ou du monastère Saint-Pantaléon de Cologne depuis au moins le Xe siècle44).

On en déduira donc que le trajet des Rogations du XIII siècle correspondait à une situation spatiale antérieure à l'érection de Xanten au rang de ville (1228), et même antérieure aux changements dans les rapports de possession à Lüttingen. Les contributions d'avouerie renvoient en effet encore à l'espace initial de l'ancienne curia archiépiscopale à Xanten au début du Xe siècle, qui fut ensuite divisée en deux cours, celle de l'archevêque et celle de la collégiale - dont l'emprise au sol locale allait former le soubassement de la future ville et dont dépendaient possessions et cours particulières à Lüttingen et Wardt.

Dans une optique "continuationniste » et plus particulièrement dans la démarche interprétative de $\mathrm{N}$. Kyll signalée plus haut, on pourrait en déduire que les litanies ne sont que les héritières de pratiques ambulatoires destinées à manifester et réaliser les liens domaniaux antérieurs au $\mathrm{X}^{\mathrm{e}}$ siècle entre une curia colonaise et ses annexes. Ceci pose bien sûr le problème classique du sens à attribuer à l'apparition somme toute tardive des plus anciennes mentions des processions (notamment des litanies) à Xanten : silence des sources (et si oui : pourquoi ?), ou absence de pratique?

Il serait sans doute risqué de considérer qu'il n'existait pas de processions antérieurement. Inversement, il faut certainement considérer d'une part la rédaction du Liber Ordinarius comme une forme de réponse à une nécessité particulière et d'autre part que cette réponse prend la forme non pas de mentions éparses mais livre d'un coup un système processionnel complet - cette structuration rationnelle, propre à la mise par écrit, constituant certainement l'apport original du Liber. Bref, si l'existence de pratiques déambulatoires ne peut certainement pas être niée pour les époques antérieures, on peut conjecturer que l'existence du système processionnel présenté auparavant ne remonte sans doute pas beaucoup plus haut que - voire est une création du Liber Ordinarius.

Ceci ne signifie pas pour autant qu'il n'y ait pas eu de critères de pouvoir temporel dans le choix des nœuds de l'espace processionnel. Simplement ces critères ne sont pas passifs, comme simples résultats d'un héritage, ils sont actifs en ce qu'ils réalisent spatialement des rapports de domination contem- 
porains. En particulier, on observe que ce n'est pas l'espace ecclésial (paroissial) mais l'espace seigneurial qui détermine le trajet des Rogations de Xanten. Bien qu'elles soient mises en œuvre par ou au nom de la hiérarchie cléricale (archevêque, prévôt, doyen, chapitre), le but des processions n'était alors possiblement pas déterminé par des critères canoniques mais par des critères temporels, seigneuriaux - en l'occurrence matérialisés par les redevances d'avouerie aux comtes puis ducs de Clèves.

Ceci pose alors de nouveau le problème du rapport entre la commune urbaine et une telle dimension spatiale. D’un côté en effet, le tribunal échevinal urbain, en tant que successeur de l'ancienne curia dominicalis de l'archevêque, étendait sa compétence judiciaire point par point sur les possessions extérieures de Xanten, dont Lüttingen et Wardt, qui devaient relever avec Fürstenberg du nouveau ressort judiciaire de Xanten constitué à la fin du Moyen Âge45. Par conséquent, les processions lors desquelles on peut supposer la présence (ou représentation) de la commune pouvaient aussi être perçues comme des démonstrations de l'extension spatiale de la compétence judiciaire des échevins. Mais d'autre part, on ne doit pas non plus négliger le fait que le seigneur de la ville était toujours un seigneur ecclésiastique et que les processions organisées et menées par la hiérarchie ecclésiastique locale ne pouvait pas ne pas apparaître en même temps comme une démonstration de la présence cléricale et seigneuriale. Les litanies auraient de ce fait revêtu un caractère ambivalent, à la fois comme moyen d'affirmation de la commune sur ses annexes et comme moyen d'affirmation du pouvoir seigneurial sur l'espace urbain.

\section{Le sens spatial du Viktorstracht}

Si le rapport entre seigneurie et identification communale dans l'espace processionnel ne peut être que déduit dans le cas des Rogations de Xanten, il est clairement à l'œuvre dans le cas de la plus célèbre procession de Xanten, le Viktorstracht (littéralement le «portement de Victor ») ${ }^{46}$. Cette procession,

45. C'est ce qu'atteste une charte de l'archevêque de Cologne pour la ville de Xanten en 1281, qui soumettait à la fiscalité urbaine non seulement le secteur de la ville proprement dite mais aussi les habitants de l'ensemble du ressort judiciaire en question : Th. Ilgen, Quellen..., op. cit., I, 2, p. $116^{*}$; K. Flink, "Zur Stadtentwicklung von Xanten (12.-14. Jahrhundert) », dans Id., Rees, Xanten, Geldern: Formen der städtischen und territorialen Entwicklung am Niederrhein, t. 1, Clèves, Boss-Verlag, 1981, p. 51. L'ouvrage le plus récent sur Xanten, celui de H. Hawicks, Xanten im späten Mittelalter. Stift und Stadt im Spannungsfeld zwischen Köln und Kleve, Cologne, Böhlau, 2007 (cf. notamment p. 133), ne se penche pas sur cet aspect.

46. D. Scheler, « Die Xantener Viktorstracht. Wallfahrt, Politik und Kommerz am Niederrhein im 15. Jahrhundert », dans Überlieferung - Frömmigkeit - Bildung als Leitthemen der Geschichtsforschung. Vorträge beim wissenschaftlichen Kolloquium aus Anlaß des achtzigsten Geburtstages von Otto 
attestée depuis 1315, n'avait lieu qu'épisodiquement, souvent à des décennies d'intervalle, normalement dans les situations de détresse, comme c'était aussi le cas pour les processions extraordinaires organisées dans la ville de Xanten, par exemple en période de peste.

La différence entre le Viktorstracht et les processions « habituelles » évoquées précédemment résidait cependant dans le fait que c'était non pas la collégiale ou la ville, mais tout l'archidiaconé, avec ses 108 églises paroissiales et ses 21 chapelles, qui devait y prendre part et mener au monastère de Fürstenberg la châsse de saint Victor accompagnée des reliques de toutes les églises qu'étaient tenus d'apporter les curés et leurs paroissiens. Et y participaient non seulement des villages mais aussi des villes régionalement importantes comme Duisbourg, Kalkar, Wesel, Rees, Emmerich et Clèves dans le duché de Clèves, Nimègue dans le duché de Gueldre ou encore Rheinberg, Kempen et Dorsten dans l'archevêché de Cologne.

Ce changement d'échelle induit un changement dans les enjeux concrets, mais pas dans leur nature - et l'on voit en effet le Viktorstracht servir à l'affirmation du pouvoir seigneurial en même temps qu'à l'affirmation des identités communales. L'affirmation à cette échelle du pouvoir seigneurial est celle du pouvoir princier, celui du comte puis duc de Clèves (définitivement maitre de Xanten depuis 1444), qui commence par être simplement représenté (par le prêt de tapisseries pour orner la collégiale, puis l'envoi des membres de sa chapelle) avant d'y apparaître en personne avec sa famille en 1464, avec tout l'apparat bourguignon auquel il avait été habitué dans sa jeunesse. Cette présence et cette coloration bourguignonne furent l'occasion d'une démonstration de grandeur du prince territorial vis-à-vis de son territoire et des princes voisins. L'archidiaconé de Xanten représentait en effet pour le prince une sorte de diocèse pour son propre territoire - et une tentative, du temps de son père, d'instauration effective d'un tel diocèse territorial, avec Xanten pour siège, avait d'ailleurs échoué, malgré l'aide bourguignonne qui lui avait pourtant permis de l'emporter face à l'archevêque de Cologne.

Mais le Viktorstracht était aussi l'occasion pour les villes et les villages de se montrer et de se faire concurrence. Les villes prirent ainsi des mesures spécifiques, par exemple en faisant confectionner des banderoles et des vêtements uniformes, en partie aux couleurs des villes concernées. Le danger que pouvaient effectivement représenter ces concurrences n'apparaît pas seulement à travers les ordonnances princières interdisant le port d'armes lors de la pro-

Meyer, J. Petersohn dir., Wiesbaden, Reichert, 1988, p. 96-113. H. Hawicks, Xanten im späten Mittelalter..., op. cit., p. 385-387, 484-485, 509-510. 
cession, mais aussi dans les négociations préalables que le doyen de Xanten mena avec les maires de Wesel et de Dorsten en 1464, afin de régler à l'avance la querelle des deux villes quant à l'ordre de préséance lors de la procession querelle qui s'était déjà par le passé soldée par des morts.

Tout ceci montre combien le Viktorstracht pouvait produire spatialement un ordre social qui dépassait largement le simple niveau ecclésial - un ordre socio-spatial dans lequel pouvaient se manifester et se réaliser non seulement les pouvoirs seigneuriaux (laïques ou ecclésiastiques) mais aussi les communes dans le cadre de ces pouvoirs. Comme dans le cas de l'espace des Rogations de Xanten, il apparaît clairement ici aussi que l'espace de l'archidiaconé n'était pas défini seulement par les délimitations ecclésiales traditionnelles mais aussi par les rapports de domination concrètement à l'œuvre. Mais bien que cet ordre instauré lors des processions soit susceptible d'être présenté comme une simple traduction spatiale de l'ordre seigneurial, il devrait bien plutôt être considéré comme ce par quoi on luttait pour s'imposer localement.

Ainsi les bourgeois de Dorsten, qui tentèrent à tout prix de s'affirmer face à ceux de Wesel, la ville la plus importante et la plus riche du duché de Clèves et de l'archidiaconé, s'appuyaient sur le fait qu'ils relevaient le plus anciennement de la collégiale, étant donné que la curia de Dorsten était entrée en sa possession dès le $\mathrm{XI}^{\mathrm{e}}$ siècle, par donation de la plus importante bienfaitrice de la collégiale, Imeza. La préséance à la procession devait ainsi permettre l'affirmation spatiale de la primauté de Dorsten, appuyée sur un ordre seigneurial que l'ordre à la procession n'était censé que révéler. Dorsten, qui ne dépendait originellement pas de l'archidiaconé de Xanten mais de celui, voisin, de Dortmund, avait d'ailleurs fini par être rattaché au plus tard au début du XIVe siècle, en une sorte d'exclave, à celui de Xanten.

L'exemple de Xanten montre donc bien, tant au niveau local que régional, qu'étudier les processions comme des indicateurs et surtout comme des facteurs de construction dynamique de l'espace de domination et de formation communautaire est une idée qui vaut la peine d'être poursuivie. C'est ce que montre en particulier le cas des Rogations, jusqu'à présent examinées au sein de leur système processionnel local mais que l'on va maintenant envisager de façon plus globale.

\section{La contribution des Rogations à la genèse d'un espace paroissial communautaire}

Envisager la contribution des processions des Rogations à la " paroissialisation » du finage pourrait sembler évident voire inutile tant le rapport entre ces processions et l'appropriation du territoire communautaire constitue un 
lieu commun de l'histoire médiévale ${ }^{47}$. Pourtant, dès que l'on commence à creuser la question, on se rend compte de la rareté des travaux sur les Rogations en général ${ }^{48}$ et sur cet aspect en particulier, négligé au même titre que la spatialité des pratiques processionnelle ${ }^{49}$, et surtout du fait que les attestations médiévales d'une telle appropriation processionnelle du finage au moment des Rogations ne sont jamais indiquées. Tout se passe alors comme si une situation effectivement connue pour la période moderne50 était rétrojetée sur la situation médiévale ${ }^{51}$. Il importe alors, pour comprendre dans quelle mesure la déambulation processionnelle des Rogations a pu constituer un facteur de construction d'un espace chrétien communautaire, de commencer à rassembler les données actuellement disponibles et de proposer quelques hypothèses.

47. Cf. entre autres A. Vauchez, "Liturgie et culture folklorique... », p. 27, 29 (151, 153); A. Guerreau, "Stabilità, via visione : le creature e il creatore nello spazio medievale », dans Arti e storia nel Medioevo, III : Del vedere : pubblici, forme e funzioni, E. Castelnuovo, G. Sergi dir., Turin, Einaudi, 2004, p. 167-197, ici p. 186-187; É. Zadora-Rio, " Territoires paroissiaux et construction de l'espace vernaculaire », Médiévales 49 (2005), p. 105-120, ici p. 117.

48. P. Collomb, «Les processions des Rogations... », p. 70, souligne pourtant l'existence d'une riche documentation liturgique (ce qui explique d'ailleurs sans doute, justement, la rareté des études historiques, puisque les documents liturgiques sont traditionnellement négligés par les historiens) - qui fondent effectivement les indications d'Emmanuel Grélois sur ClermontFerrand (travaux inédits, fondés sur l'exploitation d'un missel imprimé en 1492 et largement corroborés par une enquête de la fin du XIII' siècle [AD Puy-de-Dôme, $3 \mathrm{G}$, armoire 18, sac B, c. 32a], dont je remercie chaleureusement l'auteur d'avoir bien voulu me communiquer les résultats ; cf. aussi A. Vernière, "Itinéraires des processions dans la ville de Clermont à la fin du $\mathrm{XV}^{\mathrm{e}}$ siècle », Bulletin historique et scientifique de l'Auvergne, 6 (1886), p. 95-103), de G. Masselis sur Rouen (Processions et fêtes de rue à Rouen à la fin du Moyen Âge, ms. mémoire de Maîtrise, Rouen, 1997, notamment p. 92-94, qui s'appuie sur un ordinaire de la cathédrale de Rouen du XIV ${ }^{\mathrm{e}}$ siècle) ou encore de R. Wehner sur Wurtzbourg (Die mittelalterliche Gottesdienstordnung des Stifts Haug in Würzburg, Neustadt a.d. Aisch, Degener, 1979, notamment p. 5-50, à partir d'un ordinaire de la collégiale de Haug).

49. L'exemple de l'étude strictement temporelle et mythologique des Rogations par Ph. Walter, « L'or, l'argent et le fer... », op. cit., a déjà été signalé supra, n. 16.

50. Mais là encore, rares sont les études précises comme celle de S. Hindle, "Beating the Bounds... ", op. cit., qui montre remarquablement bien comment les Rogations ont été les seules processions à survivre à la Réformation anglaise en raison de la fonction qui leur était reconnue d'assurer la mémoire collective des limites paroissiales.

51. C'est explicitement le cas chez A. Vauchez, «Liturgie et culture folklorique... », p. 29 (153), tandis que J. Delumeau, Rassurer et protéger..., op. cit., p. 150-153, mélange des observations datant de périodes très différentes - avec comme effet de faire apparaitre le catholicisme comme un phénomène anhistorique. 


\section{Postures continuationnistes}

Dans le cas des Rogations, la posture « continuationniste » a pris deux autres formes que celle qui consiste à rétrojeter des connaissances postérieures sur la situation médiévale. D'une part, on rencontre l'idée d'une continuité entre les Rogations et certains rites de fécondité romains récupérés et christianisés, fondée sur une indéniable proximité de dates, d'usages linguistiques et de formes rituelles ${ }^{2}$. C'est ainsi que le dragon associé en certains endroits aux cortèges des Rogations a pu être considéré par certains comme le descendant des serpents associés à Rome aux cultes de fécondité53. Jacques Le Goff s'était déjà attaqué aux hypothèses de continuité romaine à propos des Rogations ${ }^{54}$, qui ne permettent en aucun cas d'expliquer la faveur de ces processions dans la société médiévale alors que tant de pratiques romaines ont par ailleurs été éliminées.

Il faut en outre souligner qu'une telle hypothèse s'accorde mal avec la distribution spatiale des mentions du dragon, qui apparaît comme un phénomène essentiellement " gallican » (c'est ce qu'affirme explicitement Jacques de Voragine, et rien ne semble devoir le contredire55), donc sans coïncidence avec l'aire d'extension des rites romains évoqués. Rappelons en outre qu'à Rome et peut-être jusqu'à la période carolingienne, c'est mars qui était le mois

52. Cf. la position classique d'A.G. Boucher d'Argis, Variété historiques, physiques et littéraires, ou recherches d'un sçavant, Paris, 1752, t. 2, p. 270, ou encore de dom de Bruyne, "L'origine des processions de la Chandeleur et des Rogations - à propos d'un sermon inédit ", Revue Bénédictine, 34 (1922), p. 14-26, qui relie les Rogations aux ambarvalia romaines, tout comme J. Heers, «Les villes d'Italie centrale et l'urbanisme : origines et affirmation d'une politique (env. 12001350) », Mélanges de l'École française de Rome - Moyen Âge, 101 (1989), p. 67-93, ici p. 74, ou encore S. Felbecker, Die Prozession. Historische und systematische Untersuchungen zu einer liturgischen Ausdruckshandlung, Altenberge, Oros, 1995, p. 194-195. On se rappellera néanmoins avoir rencontré ce terme dans le texte traduit supra, n. 27.

53. Par exemple J. Le Goff, «Culture ecclésiastique et culture folklorique au Moyen Âge : saint Marcel de Paris et le dragon ", (1970) rééd. dans Id., Pour un autre Moyen Âge. Temps, travail et culture en Occident : 18 essais, Paris, Gallimard, 1977, p. 236-279, ici p. 267-268. A. Vauchez, " Liturgie et culture folklorique... », p. 28 (153), considère également sous cet angle le rôle joué par la queue du dragon, dressée et gonflée par de la paille ou de l'étoupe les deux premiers jours, plate et pendante le troisième jour.

54. J. Le Goff, « Culture ecclésiastique... », op. cit., p. 268-269.

55. Jacques de Voragine, La Légende dorée, éd. A. Boureau, Paris, Gallimard, 2004, p. 378. De tels dragons sont en effet connus à Paris, Rouen, Vendôme, Poitiers, Bordeaux, Tarascon, Troyes, Provins, Douai, Metz, etc. J. Delumeau, Rassurer et protéger..., op. cit., p. 160, évoque également des dragons ibériques, mais ceux-ci semblent d'une part plus tardifs (mentions des $\mathrm{XV}^{\mathrm{e}}$-XVII ${ }^{\mathrm{e}}$ siècles) et surtout sans rapport avec les Rogations (Fête-Dieu). 
des serpents ${ }^{5}$, alors que les Rogations ont lieu du 27-29 mars au 31 mai-2 juin selon les années, soit après la fin de la germination, au moment de la pousse des plantes - et donc le problème serait moins celui de la fécondité stricto sensu que de la protection des futures récoltes contre les calamités (orages ou sécheresse, dégâts animaux, guerres) 57 .

L'autre face de la posture " continuationniste » concerne le rapport entre les Rogations créées au haut Moyen Âge et celles qu'on observe à partir du XII ${ }^{\mathrm{e}}$ siècle. Rappelons qu'officiellement, les Rogations ont été fondées v. 470 par l'évêque Mamert de Vienne, que cette innovation a reçu le soutien d'autres évêques, comme Sidoine Apollinaire ou Avit de Vienne (auteur d'une Homilia de rogationibus à la fin du ve siècle), puis de ceux réunis en concile à Orléans en 511, avant de s'étendre à l'Espagne au VI siècle puis à tout l'Occident selon des modalités inconnues. On ne connait cependant pas grand-chose de la pratique effective des Rogations au haut Moyen Âge, y compris à l'époque carolingienne pour laquelle on ne dispose pour l'essentiel

56. G. Comet, « Le temps agricole d'après les calendriers illustrés », dans Temps, mémoire, tradition au Moyen Âge. Actes du XIII Congrès de la SHMESP (Aix-en-Provence, 4-5 juin 1982), Aix-enProvence, PUP, 1983, p. 7-18, ici p. 11 et planche hors-texte 4.

57. Dans l'évêché d'Eichstätt, d'où provient le plus ancien registre de visites pastorales conservé pour l'espace germanique (Diözesanarchiv Eichstätt, B 230), les Rogations n'apparaissent nulle part (alors que les notices des différents villages - les villes ne sont pas visitées - mentionnent les diverses processions auxquelles se livrent les curés) : non seulement le nom lui-même est absent, mais aucune célébration ou procession n'est évoquée durant les trois jours en question (sans qu'on puisse imputer un tel silence à une absence régionale des Rogations : les Rogations sont célébrées à Wurtzbourg - cf. infra - tout comme à Bamberg : X. Haimerl, Das Prozessionswesen des Bistums Bamberg im Mittelalter, Munich, 1937, p. 9-10). Toutefois, on observe que le lendemain de l'Ascension (sans possibilité de confusion du scribe entre crastinum et vigilia, à la fois à cause de la systématicité de la mention et parce que le vendredi est parfois explicitement nommé) est très fréquemment évoqué (dans 99 paroisses sur les 307 mentionnées dans le registre) à propos d'une célébration contre la grêle (fur die schaur, fur die hagel, propter grandines), et dans 11 cas, cette célébration est explicitement accompagnée de processions (au cas où les 88 autres cas n'auraient pas connu de procession, on ne négligera pas le fait que les villageois se rendaient ce jour-là collectivement à l'église). Dans 39 cas, c'est le jour même de l'Ascension qu'a lieu une procession au cours de laquelle on circuit segetes in campis, en général cum sacramento Eucharistiae, dans quelques cas à la demande expresse des villageois. Rogations ou pas, la semaine de l'Ascension semble donc bien liée au problème de la protection des récoltes contre les intempéries (la guerre ou les parasites n'étant jamais envisagés). Les autres dates envisagées pour une telle protection des récoltes sont la Saint-Jean-et-Saint-Paul (26 juin : 58 célébrations des wetterherren, domini tonitruorum, domini aeris), la Fête-Dieu (21 mai-24 juin : 51 processions ce jour-là et 8 le lendemain ou les jours suivants, ainsi que 10 célébrations contre la grêle le lendemain ou les jours suivants), la Pentecôte (10 mai-13 juin : 44 processions et 5 célébrations), la Saint-Marc (19 célébrations contre la grêle), la Saint-Alban (21 juin : 16 célébrations contre la grêle), Pâques (22 mars-25 avril : 10 processions et 5 célébrations) et quelques autres dates d'une fréquence totale inférieure ou égale à 5 . 
que d'injonctions épiscopales théoriques ${ }^{8}$. L'examen systématique d'un ensemble de Vitae de saints mérovingiens ou carolingiens et d'annales et chroniques des IX $^{\mathrm{e}}$-XII ${ }^{\mathrm{e}}$ siècles ${ }^{59}$ montre que les Rogations sont essentiellement conçues comme une fête religieuse de référence à l'époque carolingienne et jusqu'au $\mathrm{X}^{\mathrm{e}}$ siècle, tandis que les mentions explicites et bien datées de procession intégrant le populus ${ }^{60}$ ne se rencontrent guère avant le XI ${ }^{\mathrm{e}}$ siècle. Par conséquent, même en admettant qu'il ait pu y avoir un fond pré-chrétien sur lequel les évêques gallo-romains et hispaniques auraient appuyé leur affirma-

58. Cf. supra, n. 29-30.

59. J'ai recherché systématiquement, à l'aide du moteur de recherche fourni pour la version en ligne des Monumenta Germaniæ Historica (http://www.mgh.de/dmgh/), le terme "rogatio* » (en excluant les occurrences au sens de "demande/réclamation») dans les Scriptores rerum Merovingicarum, t. 3-7, les Scriptores rerum Langobardicarum et Italicarum, les Gesta pontificum Romanorum et les Scriptores, t. 1-38 (en me limitant aux textes antérieurs à 1200, et en vérifiant la date des manuscrits utilisés). Les $\mathrm{SrM}$ présentent 7 occ., dont six signalent explicitement une célébration collective (avec populus, processio, etc.) et une un simple moment dans l'année. Il n'y a aucune occurrence dans les $S r L I$ et les $G p R$ (ni d'ailleurs dans les textes italiens, anglais et danois édités dans la série des Scriptores). Les textes allemands, flamands et du nord de la France édités dans les Scriptores présentent 58 occ., dont 40 désignent simplement un moment dans l'année, 10 une procession explicite ( 7 avec populus, 3 où seuls les moines sont mentionnés), 8 une célébration religieuse; les mentions de processions du populus remontent au plus tôt au $\mathrm{XI}^{\mathrm{e}}$ siècle (dans des manuscrits des XI ${ }^{\mathrm{e}}$-XIII ${ }^{\mathrm{e}}$ siècles), sauf pour ce qui est des Miracula de saint Wandrille, mais dont la tradition manuscrite n'est pas claire ( $\mathrm{x}^{\mathrm{e}}$ siècle ?). Dom de Bruyne, «L'origine des processions... », op. cit., p. 15, signale également un sermon du $\mathrm{X}^{\mathrm{e}}$ siècle provenant de Corbie, selon lequel « quam sollempnitatem istis rogationibus imitamur nostros circumeuntes agros, non tam pro fertilitate eorum quam pro ceteris necessitatibus animae et corporis » - mais rien ne dit que ces processions de réclamation imitant les ambarvalia correspondaient aux Rogations stricto sensu, et non pas à d'autres processions propitiatoires du même ordre et vers ces mêmes moments, comme on en rencontre par exemple massivement dans le diocèse d'Eichstätt à la fin du Xve siècle (cf. n. 57).

6o. J. Chiffoleau, «Les processions parisiennes... », op. cit., p. 41-42, rappelle qu'après l'époque carolingienne, processionner devient une affaire de moines - sans doute plus vraisemblablement de communautés religieuses (donc également canoniales). Comme on l'a vu à Xanten, l'essentiel des processions médiévales sont alors internes aux églises (l'ordinaire de SaintDenis du XIII ${ }^{\mathrm{e}}$ siècle prévoit ainsi quelque 200 processions de cet ordre chaque année: J. Delumeau, Rassurer..., op. cit., p. 94). Les processions des Rogations peuvent ainsi se dérouler également au sein de l'espace conventuel (église, cimetière, chapelles), comme semble l'indiquer le processionnal des Trinitaires de Paris (Paris, Bibliothèque Mazarine, Ms. 542, fol. 38-40). On observe également, au moment des Rogations, des processions allant des églises mères aux filiales, comme celle allant de Saint-Martin-des-Champs à Saint-Jacques-de-laBoucherie à Paris (L. Fritsch-Pinaud, « La vie paroissiale à Saint-Jacques-de-la-Boucherie au Xve siècle ", Paris et Île-de-France, 33 (1982), p. 7-97, ici p. 87) ou encore comme dans le diocèse de Gênes ou dans certains lieux de la Belgique actuelle aux XVI ${ }^{\mathrm{e}}$-XVII ${ }^{\mathrm{e}}$ siècles (U. Berlière, « Les processions des croix banales ", Bulletins de la classe des Lettres et des Sciences morales et politiques, $5^{\text {e }}$ série, 8 (1922), p. 419-446, ici p. 442) : on peut certainement y voir une extension spatiale d'un régime processionnel « interne ». 
tion locale ${ }^{61}$, le rite des Rogations a très certainement été profondément transformé (matériellement et sémantiquement ${ }^{62}$ ) entre l'époque de sa fondation et le moment où la pratique courante et de multiples commentaires théologiques apparaissent, à partir du début du XII siècle (notamment à partir du commentaire de Rupert de Deutz De rogationibus, avant 112963) : les Rogations ne se déroulent plus seulement dans les cités mais sans doute aussi dans d'autres villes ${ }^{64}$ et (à partir d'un moment inconnu) dans les campagnes ${ }^{65}$, c'est-à-dire dans l'ensemble des communautés d'habitants, où elles ne servent donc plus à la mise en scène de l'évêque (dont la présence est d'ailleurs parfois fort discrète, comme à Lyon où seul le chapitre semble représenter la cathédrale $\left.{ }^{66}\right)$.

61. Ceci correspond à la mise en place du pouvoir épiscopal à l'époque mérovingienne dans les cités de Gaule et d'Hispanie, appuyé sur la mise en scène liturgique de la dignitas épiscopale et notamment au travers du culte des reliques et des processions (cf. B. Jussen, "Liturgie und Legitimation, oder : Wie die Gallo-Romanen das Römische Reich beendeten », dans Institution und Ereignis über historische Praktiken und Vorstellungen gesellschaftlichen Ordnens, R. Blänkner, B. Jussen dir., Göttingen, Vandenhock \& Ruprecht, 1998, p. 75-136 ; B. Beaujard, « Les inventions de martyrs et l'histoire des églises de Gaule aux ve et vi siècles ", dans Autour des morts. Mémoire et identité (Actes du ve colloque international sur la sociabilité, Rouen, 19-21/11/1998), O. Dumoulin, Fr. Thélamon dir., Mont-Saint-Aignan, Publications de l'Université de Rouen, 2001, p. 363-367).

62. Cf. le cas très significatif de Schildesche signalé par L. Kuchenbuch supra.

63. De divinis officiis per anni circulum libri XII, livre IX, chap. $\mathrm{v}$ : De rogationibus a sancto Mamerto institutis, éd. J.P. Migne, Patrologia Latina, t. 170, col. 248d-250a.

64. Il importe cependant de souligner que les mentions des Rogations que j’ai repérées sont essentiellement liées aux cités (Aix, Albi, Amiens, Angers, Avignon, Bamberg, Clermont, Fréjus, Gênes, Maastricht, Marseille, Metz, Paris, Rouen, Strasbourg, Worms, Wurtzbourg). Le cas de Xanten s'explique peut-être à la fois par sa genèse épiscopale, son identité avec l'archidiaconé et sa situation "crypto-épiscopale ». Dans l'Empire, de grandes villes comme Erfurt ou Nuremberg ne semblent pas (si l'on en croit en tout cas le mutisme des travaux qui les concernent) avoir connu de processions des Rogations.

65. On observera d'ailleurs que les représentations courantes des historiens concernant les Rogations sont rurales/agricoles, alors que l'essentiel des informations que nous avons pour la période $\mathrm{XII}^{\mathrm{e}}-\mathrm{XV}^{\mathrm{e}}$ siècles sont d'origine urbaine (et qu'aucun village du diocèse d'Eichstätt ne paraît avoir connu de Rogations stricto sensu). C'est ce qui conduit A. Vauchez, "Liturgie et culture folklorique... », op. cit., p. 29 (153), à considérer comme étrange le caractère exclusivement urbain des observations de Jacques de Voragine à propos des Rogations. Indépendamment de l'argumentation habituelle en termes de sources disponibles, qui permet au moins implicitement de justifier la rétrojection sur le Moyen Âge de connaissances plus tardives, on devrait s'interroger sur les effets heuristiques d'une telle ruralisation mentale des Rogations médiévales. Le problème n'est pas seulement de se demander si le cadre villageois était bel et bien le cadre des Rogations (ce que l'exemple du Lyonnais signalé plus loin pourrait rendre contestable), mais surtout dans quelle mesure le fait de traiter séparément le cas des villes et des villages n'est pas mutilant du point de vue de notre compréhension du phénomène des Rogations (entre autres).

66. P. Collomb, « Les processions des Rogations... », op. cit., p. 85-86. 


\section{L'organisation pratique des Rogations}

Ceci impose alors d'examiner plus nettement les aspects pratiques du rituel. On ne s'étonnera évidemment pas de constater que les Rogations sont toujours organisées par les clercs, puisque processionner est avant tout un rituel clérical (les clercs processionnant entre eux, devant les fidèles ou non). Mais en ce qui concerne les Rogations, pourtant censées assurer l'appropriation communautaire de l'espace, on observe que dans de multiples cas, les clercs étaient officiellement les seuls à défiler devant le public laïque : c'était le cas à Lyon comme à Marseille et à Rouen ${ }^{67}$, et même les données fournies par Jacques de Voragine (qui évoque la « multitude des fidèles » suivant la procession ${ }^{68}$ ) ne sont pas claires quant au rôle exact des fidèles. Suivre la procession, voire même y assister de loin comme à Lyon où les fidèles suivent sur les berges de la Saône le retour de la procession le deuxième jour69, est-ce y prendre part ? Jacques Chiffoleau, distinguant participation physique et spectacle ${ }^{70}$, le nierait sans doute, mais cette opposition repose largement sur une notion du « voir » qui nous est propre et dont l'emploi pour la période et/ou la pratique processionnelle est désormais contesté ${ }^{11}$.

Tout se passe donc comme si les Rogations étaient en premier lieu une procession de l'église militante (le clergé) devant le reste du corps ecclésial (les fidèles) - ce " devant » ayant sans doute signifié « face à » avant de devenir " à la tête de » aux XIVe-XVe siècles, mais sans que ce « face à » exclue une participation effective, de la même manière que lors de l'élévation pendant la messe. Il faudrait alors considérer les processions non seulement comme une manière de marcher ensemble, mais aussi comme une assistance commune (comme c'est aussi le cas dans les processions municipales lors desquelles la ville s'auto-représente). La procession instaure ainsi un rapport entre ceux qui marchent et ceux qui ne marchent pas (seul moyen de donner un sens so-

67. Ibid., p. 90-91 ; N. Coulet, « Processions... », op. cit., p. 392 ; G. Masselis, Processions..., op. cit., p. 92 (les seuls laïcs participants étant les membres des confréries).

68. J. de Voragine, La légende dorée, op. cit., p. 378.

69. P. Collomb, « Les processions des Rogations... », op. cit., p. 72.

70. J. Chiffoleau, « Les processions parisiennes... », op. cit., p. 72-74.

71. Sur la spécificité médiévale du « voir », qui 1) implique une participation active de celui qui voit et 2) repose sur une distinction entre la perception sensible et l'approximation spirituelle, voir B. Duden, I. Illich, « Die skopische Vergangenheit Europas und die Ethik der Opsis. Plädoyer für eine Geschichte des Blickes und Blickens ", Historische Anthropologie, 3/2 (1995), p. 203-221; C. Havelange, De l'œil et du monde. Une histoire du regard au seuil de la modernité, Paris, Fayard, 1998 ; A. Guerreau, "Stabilità, via visione... », op. cit. Dans le cas particulier des processions, voir S. Felbecker, Die Prozession..., op. cit., p. 524-529, et H. Kühne, "Ostensio reliquiarum ». Untersuchungen über Entstehung, Ausbreitung, Gestalt und Funktion der Heiltumsweisungen im römisch-deutschen Regnum, Berlin/New York, De Gruyter, 2000, p. 828-832. 
cial au fait de marcher), tout comme le fait l'organisation temporelle de la procession entre les moments de marche et les moments de station ${ }^{72}$.

Le rapport clercs/laïcs est également activé à travers le culte des saints auquel sont explicitement liées les Rogations (Jean Beleth écrit clairement : rogatio appellatur, quia tunc sanctos rogamus, ut pro nobis ad Dominum intercedant73), ce que développe largement Jacques de Voragine ${ }^{74}$, alors que le clergé s'est efforcé de contrôler le culte des saints. Les Rogations consistent même en une invocation "de tous les saints » (Jacques de Voragine) ${ }^{75}$, ce qui renvoie à la conception de la " communion des saints ", qu'est censé n'assurer que le seul clergé. Dans cette perspective, les Rogations pourraient apparaitre comme un rituel complémentaire à celui de la messe (où l'ecclesia entre en contact avec dieu par le biais de sa partie active, le clergé) et à celui du pèlerinage (où les chrétiens entrent personnellement en contact avec un saint particulier).

Toutefois, la distinction entre contact avec dieu et contact avec les saints ne devrait cependant pas être surévaluée, à la fois parce que les saints sont de toute façon et seulement des intercesseurs ou médiateurs nécessaires (les églises sont dans leur immense majorité dédiées à des saints, et non au saint Sauveur ou au saint Esprit, et il doit obligatoirement y avoir des reliques dans l'autel) et parce que le déplacement des reliques des saints lors des Rogations se déroule dans un cadre strictement christologique (il s'agit des trois jours précédant l'Ascension). Quant à la distinction entre pèlerinage et procession, elle est loin d'être aussi évidente, du point de vue historique, qu'il y paraît ${ }^{7}$. Il est alors possible que, du point de vue

72. S. Felbecker, Die Prozession..., op. cit., p. 385-389.

73. Jean Beleth, Rationale divinorum officiorum, éd. J.P. Migne, Patrologia Latina, t. 202, col. 9$166 \mathrm{c}$, ici col. 128c.

74. J. de Voragine, La légende dorée, op. cit., p. 378-379.

75. Ibid., p. 376, 378.

76. Le lien plus qu'étroit entre la procession et le pèlerinage médiévaux a fait l'objet d'un intense débat en Allemagne parmi les ethnographes, à la suite des travaux de H. Dünninger, "Processio peregrinationis ». Volkskundliche Untersuchungen zu einer Geschichte des Wallfahrtswesens im Gebiet des heutigen Diözese Würzburg, Würzburg, Bischöfliches Ordinariatsarchiv (Würzburger Diözesangeschichtsblätter, 23/24), 1961/1962, qui a souligné l'emploi du même verbe (wallen) dans les deux cas et l'usage indifférent des termes walfart, zulauf, kriuzfart. Ces résultats obtenus pour la Franconie ont été élargis à la Bavière et à la Rhénanie par W. Brückner, «Zur Phänomenologie und Nomenklatur des Wallfahrtswesens und seiner Erforschung. Wörter und Sachen in systematisch-semantischem Zusammenhang », dans Volkskultur und Geschichte. Festgabe für Josef Dünninger zum 65. Geburtstag, D. Harmening et alii dir., Berlin, E. Schmidt, 1970, p. 384-424. Plus récemment encore, R. Tekippe, «Pilgrimage and Procession : Correlations of Meaning, Practice, and Effects ", dans Art and Architecture of Late Medieval Pilgrimage in Northern Europe and the Britisch Isles, S. Blick, R. Tekippe dir., Leyde, Brill, 2004, p. 693-752, ici p. 694, 696, a réaffirmé la difficulté voire l'impossibilité de distinguer, dans la documentation médié- 
théologique voire liturgique, il s'agisse plutôt d'un redoublement que d'une complémentarité.

En revanche, il y a bien complémentarité du point de vue spatial. Les Rogations sont en effet expressément conçues comme un déplacement de reliques d'un sanctuaire à l'autre au sein d'une même ville77. Et la même chose s'observe peut-être aussi dans les campagnes si l'on en croit l'exemple du Lyonnais aux XIVe-XVe siècle : les testaments révèlent qu'au moment des Rogations, les processions associent les églises paroissiales (avec leurs reliques) de Saint-Symphorien-sur-Coise, Pomeys, Coise et Larajasse (au sud-ouest de Lyon) ; une telle symbiose de paroisses s'observe aussi dans les Monts du Tarare (à l'ouest de Lyon) et dans le Jarez (région au sud-sud-ouest de Lyon, à l'ouest de Vienne) ${ }^{78}$.

Les Rogations sont ainsi bien un phénomène inverse (ou complémentaire) à celui du pèlerinage et de la messe, puisque ici ce sont les reliques qui sortent des églises et se déplacent, tandis que dans les autres cas, ce sont les fidèles

vale, entre pèlerinage et procession ; voir également J. Gengnagel et alii, « Einleitung », op. cit., p. 3, 11. La distinction entre les deux est donc tardive et repose sur l'application à ce phénomène d'une grille de lecture opposant l'individuel et le collectif ou encore, comme l'a souligné G. Signori, «Ereignis und Erinnerung... », op. cit., p. 107, le privé et le public - seul le rite collectif étant censé faire advenir, dans les sociétés anciennes, l'identité collective.

77. La Vita de saint Ouen signale ainsi le déplacement des reliques de saint Ouen au moment des Rogations à Rouen, entre l'abbaye de saint Ouen et la chapelle Saint-Michel (P.-A. Sigal, L'homme et le miracle..., op. cit., p. 159), et G. Masselis, Processions..., op. cit., p. 92, mentionne également les châsses de la Vierge et de saint Romain ainsi que tous les reliquaires du trésor métropolitain. À Paris au plus tard v. 1200, c'est le reliquaire de la Vierge qui est transporté le lundi, jusqu'à Montmartre (A. Friedmann, Paris, ses rues, ses paroisses du Moyen Âge à la Révolution. Origine et évolution des circonscriptions paroissiales, Paris, Plon, 1959, p. 410). À Lyon du XIII ${ }^{\mathrm{e}}$ au XVIII ${ }^{\mathrm{e}}$ siècle et sans doute déjà au XII ${ }^{\mathrm{e}}$ siècle, des clercs portent plusieurs châsses avec des reliques provenant de la cathédrale Saint-Jean, de l'église Saint-Paul et d'autres églises encore (P. Collomb, "Les processions des Rogations... », op. cit., p. 83, 87-88). À Marseille, au plus tard au XIII siècle, on va chercher les reliques à l'église Saint-Cannat le mercredi, on les amène à la cathédrale où la châsse est placée de telle manière que les fidèles passent au-dessous en entrant et en sortant de la cathédrale (où a lieu un office), puis elles sont ramenées à SaintCannat (N. Coulet, "Processions... », op. cit., p. 390). À Aix-en-Provence avant le milieu du $\mathrm{XIV}^{\mathrm{e}}$ siècle, on amène au moment des Rogations "les reliques aux lieux habituels " (ibid., p. 392). À Wurtzbourg, le bréviaire de la cathédrale signale que les chanoines de Haug apportent des reliques à la cathédrale (R. Wehner, Die mittelalterliche Gottesdienstordnung..., op. cit., p. 7).

78. M.-T. Lorcin, « Les clauses religieuses dans les testaments du plat pays lyonnais aux XIve et XVe siècles ", Le Moyen Âge, 78 (1972), p. 287-323, ici p. 302. L'examen du registre de visites pastorales du diocèse d'Eichstätt déjà mentionné fait apparaître, en dépit de l'absence des Rogations stricto sensu, des célébrations apparentées du point de vue agraire (cf. supra, n. 57), dont plusieurs processions explicitement conçues comme reliant plusieurs églises (cf. à Eutenhofen [fol. 24v] : Dixit quod crastinum Ascensionis venerentur fur di hagel et ipse plebanus ipsa die cum vexillis absque sacramento circuiat segetes de una ecclesia ad alteram ; à Schwabach [fol. 83v], il est dit de façon plus allusive : Crastinum Ascensionis, parochiani extra parochiam venerantur fur di schaur). 
qui se déplacent pour aller aux reliques. Ceci s'observe certes dans d'autres processions (pour conjurer un malheur), mais ce qui en distingue les Rogations, c'est que la sortie des reliques est acceptée par le clergé de l'église détentrice alors que dans les autres cas, il freine des quatre fers avant de prêter ses reliques.

Les Rogations apparaissent ainsi comme une pratique processionnelle dotée d'un sens propre et spécifique, non réductible au seul déplacement des reliques (commun à toutes les processions) mais qui réside sans doute dans la manière dont on les déplace, notamment par la mise en réseau des églises locales. En effet, de même que les Rogations tendent à constituer l'église militante (le clergé qui processionne), elles réalisent aussi l'union des églises locales via leurs reliques. D'où l'insistance sur le caractère homogène de la procession, qui se traduit par exemple à Lyon par l'interdiction formelle d'interrompre le cortège 79 .

Les Rogations apparaissent ainsi comme un rituel qui permet de dépasser le « polycentrisme religieux » (J. Chiffoleau) qui caractérise nettement les vil$\operatorname{les}^{80}$. Le cas des campagnes lyonnaises des XIVe-XVe siècles mériterait d'être réexaminé plus précisément, notamment pour voir si les diverses églises associées ne résultent pas du démembrement antérieur d'une macro-paroisse carolingienne ${ }^{81}$, avant la réduction du trajet à la seule paroisse villageoise puisque aussi bien à l'époque moderne, il semble que le trajet "classique » ait été le suivant : le lundi (et mardi), un circuit au départ de l'église paroissiale et conduisant dans les diverses chapelles et oratoires du terroir; le (mardi et) mercredi, un circuit au départ de l'église et allant jusqu'aux limites du finage dont on fait le tour ${ }^{82}$. Mais ce qui est significatif, c'est que ce dépas-

79. P. Collomb, «Les processions des Rogations... », op. cit., p. 90. C'est peut-être ainsi qu'il faut aussi entendre la présence à Rouen de curés armés de baguettes ad defendendam et custodiendam processionem (G. Masselis, Processions..., op. cit., p. 92).

80. J. Chiffoleau, « Note sur le polycentrisme religieux urbain à la fin du Moyen Âge », dans Religion et société urbaine au Moyen Âge. Études offertes à Jean-Louis Biget, P. Boucheron, J. Chiffoleau dir., Paris, Publications de la Sorbonne, 2000, p. 227-252.

81. Ce modèle de la subdivision de l'Urpfarrei (la plebs, la pieve, la plou, etc.) du haut Moyen Âge et dont auraient résulté les paroisses de la fin du Moyen Âge est désormais contesté (cf. notamment M. Lauwers, "Paroisse, paroissiens et territoire. Remarques sur parochia dans les textes latins du Moyen Âge », Médiévales 49 (2005), p. 11-32), non pas dans le principe d'une démultiplication des églises paroissiales à partir de quelques églises-mères mais parce qu'il repose sur l'acceptation implicite de l'existence dès le haut Moyen Âge de territoires paroissiaux - bref parce qu'il néglige que la démultiplication des églises et des ressorts paroissiaux s'est accompagnée d'une transformation des rapports socio-spatiaux.

82. A. Vauchez, «Liturgie et culture folklorique... », op. cit., p. 27 (151). En Angleterre, l'évolution est présentée dès 1560 par l'évêque de Londres comme le passage d'une deambulation à une perambulation (au sens ancien de déplacement périphérique), quoique au départ de l'église paroissiale : S. Hindle, "Beating the bounds... », op. cit., p. 208. Dès la fin des années 
sement des polycentrismes prenne la forme d'une déambulation et ce (mis à part le cas des campagnes lyonnaises - ou encore celles du diocèse d'Eichstätt) dans le cadre du finage de la communauté d'habitants.

\section{Examen des itinéraires}

Contrairement aux processions conjuratoires conjoncturelles, les itinéraires empruntés par les processions des Rogations sont considérés comme particulièrement durables. Ils ne sont pas toujours connus précisément : à Aix, on se contente ainsi de mentionner au milieu du XIVe siècle les « lieux accoutumés » ${ }^{8}$ - mais justement, cette mention d' « accoutumés » montre que les choses sont ancrées dans une sorte de tradition. À Lyon, Pascal Collomb observe ainsi une continuité régulière et parfaite du XII siècle jusqu'au début du XVII ${ }^{e}$ siècle : " le parcours est intangible, les oraisons et les pièces processionnelles le sont également » ${ }^{84}$. C'est pourquoi à Avignon, la reconstitution par Marc Venard du trajet processionnel de l'époque moderne fait apparaitre qu'il est étroitement fidèle au plan de la ville d'avant 1226, ce dont il déduit que le trajet de l'époque moderne est celui qui s'est mis en place v. $1200^{85}$. De même à Angers, les Rogations ne se déroulent que sur la rive gauche de la Maine (quoique dans l'espace enclos au XIII ${ }^{\mathrm{e}}$ siècle), donc à l'exclusion du bourg apparu sur la rive droite au XI ${ }^{\mathrm{e}}$ siècle ${ }^{86}$ - et l'on a vu aussi qu'à Xanten, le trajet des Rogations du XIII siècle paraît remonter à une situation spatiale du $\mathrm{x}^{\mathrm{e}}$ siècle.

D'une manière générale, on observe d'ailleurs que les couvents Mendiants sont à l'écart des itinéraires en question (sauf lorsque ceux-là sont fondés là où ceux-ci passaient déjà), ce qui suggère que ces itinéraires sont antérieurs

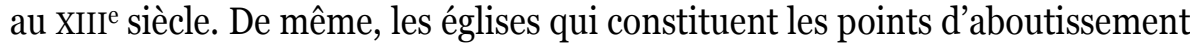
des processions journalières (Montmartre à Paris, Saint-Serge, Saint-Aubin et le prieuré de l'Évière à Angers, Chantoin et Chamalières à Clermont, Lüttingen/Wardt, Fürstenberg et Hagenbusch à Xanten, Saint-Privat et SaintQuentin à Metz, etc.) constituent l'entourage ecclésiastique des villes en question au plus tard au XII siècle.

1470, toutefois, les marguilliers de Tilney (Norfolk) désignent les processions des Rogations comme une perambulacion (ibid., p. 214).

83. N. Coulet, « Processions... », op. cit., p. 386 (portando reliquias per loca consueta).

84. P. Collomb, « Les processions des Rogations... », op. cit., p. 70.

85. M. Venard, «Itinéraires de processions... », op. cit., p. 61.

86. J.M. Matz, « Le développement tardif... », op. cit., p. 355-356. 
Lorsqu'on dispose de l'itinéraire complet (Lyon, Rouen, Angers, Wurtzbourg et d'une certaine manière Marseille), on observe un itinéraire qu'on pourrait appeler « polycirculaire rayonnant » : à partir de la cathédrale, on se dirige chaque jour vers une extrémité du territoire de la ville (d'où « rayonnant $)^{87}$ selon un itinéraire en boucle ${ }^{88}$, comme le montrent le plan de Wurtzbourg et les croquis en annexe ${ }^{89}$. La même chose s'observe à Clermont comme à Metz, où l'on ne connaît le trajet que pour deux jours ${ }^{90}$. À Paris, on ne connaît que le trajet de la première journée des Rogations ${ }^{91}$, qui présente avec celui de Metz et de Lyon le point commun d'aboutir à une colline extramuros, surplombant la ville, mais relevant toujours du territoire de la ville : Montmartre au nord de Paris, le Mont-Saint-Quentin à l'ouest à Metz, la colline de Saint-Irénée à Lyon (c'est la même chose, cette fois le mercredi, à

87. P. Collomb, «Les processions des Rogations... », op. cit., p. 75, parle pour Lyon d'un itinéraire " en étoile »; cf. aussi le trajet à Xanten décrit supra. J. Delumeau, Rassurer et protéger..., op. cit., p. 150-153, présente de tels itinéraires rayonnants (tardifs), mais qu'il considère plutôt comme un mode de «quadrillage » (métaphorique, et non pas géométrique) de l'espace communal.

88. M. Venard, «Itinéraires de processions... », op. cit., p. 60, rappelle opportunément que « les points extrêmes ne sont pas les buts ", puisque les points de départ et d'arrivée sont identiques, à savoir la cathédrale. La notion d' « étoile » risquant de faire oublier cet aspect du retour, il est donc préférable de l'écarter.

89. Ces croquis appellent deux remarques : 1) ils sont fondés sur des plans déjà publiés (par J.M. Matz pour Angers, P. Collomb pour Lyon, N. Coulet pour Marseille - mais sans l'itinéraire détaillé -, G. Masselis pour Rouen), à l'exception de Clermont (qui repose sur un plan inédit détaillé d'E. Grélois : cf. supra, n. 48) et de Wurtzbourg (plan que j’ai moi-même établi à partir du travail de R. Wehner, Die Gottesdienstordnung..., op. cit.). 2) N'y figure que la procession générale, alors qu'existaient également des processions "secondaires ", concernant des établissements particuliers, soit "greffées » sur la procession principale (p. ex. à Lyon : le mardi, le clergé de la collégiale Saint-Just revient par un autre itinéraire - représenté par P. Collomb que celui du reste de la procession, qui rentre par la Saône), soit apparemment indépendantes (p. ex. la procession parisienne allant de Saint-Martin-des-Champs à Saint-Jacques-de-laBoucherie le mardi : L. Fritsch-Pinaud, « La vie paroissiale... », op. cit., p 87).

90. Clermont : cf. supra, n. 48. Metz : M. Chazan, « Les processions à Metz », dans Le chemin des reliques. Témoignages précieux et ordinaires de la vie religieuse à Metz au Moyen Âge, Metz, Musée de la Cour d'Or, 2000, p. 122-126, ici p. 122 (je remercie l'auteure de m'avoir procuré ce texte) : on ne connait pas le trajet précis, mais seulement les deux points d'aboutissement du premier et du troisième jour.

91. Au départ de la cathédrale, il aboutissait à Montmartre (cf. supra, n. 77), par un trajet inconnu mais apparemment direct, donc sans doute par les portes Montmartre ; il en repartait ensuite pour Saint-Lazare puis Saint-Laurent, entrait dans Paris par la porte Saint-Martin, passait par Saint-Merry avant de parvenir à la cathédrale, si l'on en croit les indications fournies en 1427 par le Journal d'un bourgeois de Paris de 1405 à 1449, éd. C. Beaune, Paris, Librairie Générale Française, 1990, p. 230-231. 
Rouen et à Wurtzbourg)92. On a ainsi le sentiment que le premier ou (dans le cas de Lyon et d'Avignon) les deux premiers jours permettent d'inclure la banlieue dans l'itinéraire ${ }^{93}$, alors qu'à Marseille, on ne sortait pas des murs...

Les itinéraires en question pourraient être crédités d'une double fonction. D'une part, dans une perspective d'histoire des mentalités quelque peu déshistorisée, ils peuvent être considérés comme des "rites de clôture », l'établissement d'un « cercle magique » (Noël Coulet)94 protecteur qui mobilise à la fois le sens prêté à la figure du cercle et les représentations martiales qui peuvent être développées à propos des Rogations95. Mais cette clôture n'a sans doute pas (seulement) un sens apotropaïque : elle sert à démarquer la communauté des communautés voisines, en allant jusqu'aux églises liminaires comme on l'observe à Lyon, Rouen, Avignon, Metz ou encore Paris (Montmartre est ainsi conçue comme un point-limite entre Paris et SaintDenis $\left.{ }^{96}\right)$.

D'autre part, ils tendent à homogénéiser le territoire de la communauté en question, au-delà des divisions courantes : à Marseille, la ville haute, épiscopale, et la ville basse, vicomtale puis communale ; à Lyon, les deux rives de la Saône, c'est-à-dire aussi la cité épiscopale, à l'ouest, et la ville communale à l'est ; la même chose peut être dite pour Paris (la Cité et la Ville), Wurtzbourg

92. Cette « ascension » d'une colline surplombante, à partir de laquelle tout le finage peut être béni, semble constituer (lorsque la topographie le permet) un élément non seulement systématique mais également structurant du rituel, si l'on en croit les exemples plus tardifs et les analyses ethnographiques qu'en rappelle J. Delumeau, Rassurer et protéger ..., op. cit., p. 152.

93. À Avignon, la procession sortait dans la campagne le lundi (jusqu'à la chapelle NotreDame-de-Bonne-Aventure, au nord-est) et le mardi (au monastère de Saint-Ruf, au sud de la ville), mais le mercredi, on restait dans la ville : M. Venard, « Itinéraires de processions... », op. cit., p. 60, 62. Toutefois, dans la mesure où le point extrême de la procession du mercredi se trouvait au-delà de la muraille du XII ${ }^{\mathrm{e}}$ siècle, on ne peut exclure qu'avant la construction de celle du XIve siècle, les trois itinéraires avaient tous englobé la banlieue.

94. "Cercle magique » dont un des caractères serait qu'il tourne dans le sens contraire des aiguilles d'une montre : N. Coulet, "Processions... ", op. cit., p. 391, qui renvoie à M. Venard, "Itinéraires... », op. cit., [p. 6o], ce que constate aussi à Toulouse Fr. Bordes, «Une perception... ", op. cit., p. 140. Mais divers contre-exemples peuvent être avancés, comme le mardi à Angers, le lundi à Clermont, le mardi et le mercredi à Lyon, les trois jours à Rouen et à Wurtzbourg, si bien que le sens observé à Avignon, Marseille et Toulouse a toutes les chances d'être un hasard. Le lien entre rites de clôture (itinéraires ronds, fermés) et protection est aussi admis par principe par J. Chiffoleau, « Les processions parisiennes... », op. cit., p. 42-43, 53.

95. J. de Voragine, La Légende dorée, op. cit., p. 377-378, assimile le cortège processionnel à une armée, ce que fait plus explicitement encore l'évêque et liturgiste Guillaume Durant, dans son Rationale divinorum officiorum composé avant 1291 (cf. J. Delumeau, Rassurer et protéger..., op. cit., p. 114-115, 121).

96. A. Lombard-Jourdan, «Montjoie et saint Denis ! ». Le centre de la Gaule aux origines de Paris et de Saint-Denis, Paris, CNRS, 1989, p. 193-200. 
(la ville et le Marienberg97), Clermont (la cité et le bourg Saint-Alyre98)... De la même manière et surtout, les Rogations transcendent les « limites " paroissiales ${ }^{99}$ - dont l'origine est seigneuriale bien plus que canonique, on l'a dit. Bref, les itinéraires semblent instituer une hétérotropie (endroits dotés de valeurs particulières) qui se surimpose à et masque l'organisation profane de l'espace.

Qui plus est, l'horizon théorique logique de cette homogénéisation est celui de la chrétienté latine : cérémonie liée au cycle pascal, donc dotée d'une date unique dans tout l'Occident chrétien, les Rogations mettaient alors en marche le même jour, dans tout l'Occident, en ville comme au village, selon un mouvement par conséquent à la fois général et pluri-local, invocateur du même peuple des saints donc s'insérant dans une même fraternité spirituelle, l'ensemble des églises locales suivies par leurs fidèles ${ }^{100}$ - ce qui permet sans doute, en certains endroits, aux processions de se rencontrer et donc de ritualiser la démarcation qui deviendra semble-t-il un élément clé des Rogations, en tout cas à la campagne ${ }^{101}$.

$\mathrm{Au}$-delà de la référence idéale à la chrétienté peut-être plus nettement marquée dans les Rogations que dans les autres processions (mais qui sera aussi très présente dans les processions de la Fête-Dieu à partir de la fin du XIII siècle), la spécificité des Rogations est en effet leur articulation particulière avec l'organisation des communautés d'habitants. D'une part, tous les cas examinés (y compris celui de Xanten) montrent que la spécificité des Rogations est qu'elles ne se restreignent en aucun cas au cadre paroissial, pas plus en ville (où il existe de multiples paroisses) qu'à la campagne, comme le

97. La colline du Marienberg, à l'ouest de la ville et de l'autre côté du Main, est l'endroit où l'évêque de Wurtzbourg a installé sa résidence en 1253, après une émeute urbaine.

98. Sur le caractère de « ville double » de Clermont/Saint-Alyre (Saint-Alyre est un bourg monastique extra-muros doté d'une justice territoriale propre), cf. E. Grélois, Territorium civitatis. L'emprise de l'Église sur l'espace d'une cité et de ses environs : Clermont au XIII siècle, ms. thèse, Paris I, 2003, t. 2, p. 445-457.

99. Soit parce que le trajet traverse les divers ressorts paroissiaux (à Aix-en-Provence, toutes les paroisses sont ainsi concernées : M. Venard, «Itinéraires... », op. cit., p. 58), soit parce que toutes les églises paroissiales doivent être représentées lors des processions, comme le signale bien l'acte de fondation de la paroisse Saint-Jean-en-Grève de Paris, en 1213 (cf. supra, n. 77).

100. A. Guerreau, «Stabilità, via visione... », op. cit., p. 186. Il est donc rigoureusement logique d'observer que Jacques de Voragine argumente en termes de "l'église ", «les fidèles", «nous ", « on », « chacun ». Le cas du diocèse d'Eichstätt signalé plus haut, où les Rogations (rurales) sont absentes, contraint toutefois à remettre en cause cette belle unanimité, qui pourrait n'être que le résultat d'une normalisation tardive.

101. Pour ce qui est de l'Angleterre moderne, S. Hindle, « Beating the bounds... », op. cit., p. 219, signale de tels cas de rencontres. 
montre l'exemple des villages lyonnais mentionnés antérieurement. Ce dernier cas (qu'il conviendrait cependant de creuser) montre en outre que la corrélation entre Rogations et communautés d'habitants n'allait pas d'emblée de soi, puisque les processions semblent pouvoir avoir concerné plusieurs finages.

Mais on devrait alors rappeler les observations faites par Samuel Leturcq à propos de la châtellenie sandionysienne de Toury, où l'on observe l'existence de plusieurs échelles (du hameau au groupe de paroisses en passant par le village proprement dit, constitué par le bourg et les hameaux qui en dépendent $^{102}$ ) d'appropriation communautaire spécifique des ressources matérielles et sociales, en quoi l'on devrait voir ce qui fonde un territoire ${ }^{103}$. La question qu'il convient alors de régler est celle du rapport entre le village et la communauté d'habitants rurale, que l'on tend à considérer comme équivalents ${ }^{104}$. Mais si l'on considère comme déterminant l'enracinement (l'habitat) dans un espace d'appropriation collective de ressources (terres arables, champs moissonnés, incultes, hydrologie naturelle ou artificielle, mais aussi, en ville, accès à la production et à la commercialisation artisanales), alors il devient périlleux de se focaliser sur le village proprement dit, qui privilégie la morphologie sur les rapports socio-spatiaux ${ }^{105}$.

Une autre spécificité des Rogations par rapport aux autres processions est l'existence des dragons processionnels, qui n'apparaissent selon toute vraisemblance pas avant le XII siècle ${ }^{106}$. L'étude de J. Le Goff l'a conduit à observer, une fois écartée l'idée d'un héritage romain, que « la seconde moitié du XII et le XIII siècle voient se développer dans l'Occident chrétien une emblématique urbaine du dragon [...], qui devient l'emblème protecteur de la

102. S. Leturcq, «Territoires agraires... », op. cit., et « Espace du village, terrouers des hameaux. Théories et pratiques spatiales d'une communauté paysanne en Beauce orléanaise aux XIV et $\mathrm{XV}^{\mathrm{e}}$ siècles », dans Construction de l'espace au Moyen Âge..., op. cit., p. 229-241.

103. L. Assier-Andrieu, « La communauté villageoise. Objet historique. Enjeu théorique », Ethnologie française, 1986, p. 351-360.

104. L. Assier-Andrieu, ibid., p. 359, considère ainsi comme équivalents communauté villageoise, commune agricole, commune rurale, collectivité agro-pastorale, communauté locale, communauté d'habitants, etc. C'est par rapport à ce paradigme \{village = communauté d'habitants par excellence\} que se comprend la notion de « village éclaté » de D. Pichot, Le village éclaté. Habitat et société dans les campagnes de l'Ouest au Moyen Âge, Rennes, PUR, 2002.

105. É. Zadora-Rio, « Le village des historiens et le village des archéologues », dans Campagnes médiévales : l'homme et son espace. Études offertes à Robert Fossier, É. Mornet dir., Paris, Publications de la Sorbonne, 1995, p. 145-153, rappelle fort opportunément les écarts heuristiques, sur un même objet social, liés à l'appréhension soit de l'une, soit des autres.

106. J. Le Goff, « Culture ecclésiastique... », op. cit., p. 269-275; J. de Voragine, La légende dorée, op. cit., p. 1237. Des travaux de P. Collomb sur les dragons processionnels sont en cours. 
ville », parce qu'il incarne essentiellement le monde sauvage, brut, barbare, le monde du limon primitif dont la soumission signifie symboliquement l'accession de la ville au monde cultivé et civilisé ${ }^{107}$. Cette dimension symbolique expliquerait alors pourquoi il n'y a pas de dragons ruraux ${ }^{108}$ : les campagnes ne se définissent pas par une telle distinction (et elles sont même régulièrement assimilées par les citadins au monde sauvage et boueux).

Toutefois, cela n'explique pas pourquoi seul l'espace " gallican » semble concerné. Mais surtout, si le dragon, une fois dompté, devient une sorte de " mascotte » de la ville ${ }^{109}$, et si les Rogations sont des processions spécifiquement organisées par le clergé, alors on doit se demander dans quelle mesure les dragons processionnels ne sont pas une manifestation symbolique de la soumission de la ville au pouvoir protecteur de l'Église : à Lyon, il est clair que les Rogations doivent être considérées comme une forme d'affirmation du pouvoir clérical face au pouvoir municipal (soutenu par le roi), de même que «l'invasion » cléricale de la partie vicomtale puis communale de la ville de Marseille ${ }^{110}$. En ce sens, le fait même que les itinéraires des trois jours fassent se succéder des déambulations intra-muros et des sorties hors-les-murs doit sans doute être considéré comme une façon de montrer le caractère tout relatif des murailles ${ }^{111}$ dont on sait par ailleurs l'importance dans la prise de conscience communautaire et la formation des pouvoirs municipaux ${ }^{112}$.

107. Ibid., p. 255-258 (le dragon comme signe de la nature sauvage, dont le domptage exprime le processus de civilisation - chrétienne), 274 (passage cité) ; A. Vauchez, « Liturgie et culture folklorique... ", op. cit., p. 28 (152).

108. Ce qu'observait aussi A. Vauchez, «Liturgie et culture folklorique... », op. cit., p. 29 (153), mais qu'il expliquait par le fait (purement psychologique...) que « le paysan ne pouvait se permettre, à la différence du citadin, de se moquer des bêtes qu'il redoutait et dont il avait besoin. »

109. Ibid., p. 28 (152) ; J. Le Goff, « Culture ecclésiastique... », op. cit., p. 273.

110. P. Collomb, «Les processions des Rogations... », op. cit., p. 77, 84 ; N. Coulet, « Processions... », op. cit., p. 391.

111. De même que le sens des processions se construit par rapport au fait de ne pas marcher (cf. supra, n. 72), le sens de la sortie extra-muros se construit par rapport au fait de rester intramuros - donc la sortie hors les murs durant deux des trois jours comme une forme de négation de la clôture. Le cas marseillais devrait de ce fait être réexaminé.

112. Voir par exemple le Corpus des sceaux français du Moyen Âge, t. 1 : Les sceaux des villes, éd. B. Bedos, Paris, Archives Nationales, 1980 : sur 715 sceaux lisibles (en laissant de côté les contre-sceaux) répertoriés entre la fin du XII ${ }^{\mathrm{e}}$ siècle et 1500 pour la France actuelle, 167 représentent un élément de fortification (enceinte, porte fortifiée, tour, etc. ; peut-on y ajouter 7 représentations de clefs ?), 145 un écu armorié, 96 des saints, la Vierge ou l'agneau pascal, etc. ; mais on ne rencontre qu'un seul beffroi et 3 ou 4 hôtels de ville. Ả ma connaissance, l'étude précise du corpus n'a pas été faite. Pour l'Allemagne, voir entre autres A. Nitschke, « Die Mauern werden höher. Zum bürgerlichen Selbstverständnis im Mittelalter », Die alte Stadt. Zeitschrift für Stadtgeschichte, Stadtsoziologie und Denkmalpflege, 16 (1989), p. 327-338 (réimp. dans Id., Fremde Wirklichkeiten, II, Goldbach, Keip, 1995, p. 193-204); H. Koller, " Die mittelalterliche 
On remarquera que Rupert de Deutz ne parle pas des dragons dans son $D e$ Rogationibus, au contraire du liturgiste Jean Beleth un demi-siècle siècle plus $\operatorname{tard}^{113}$, comme si le thème de la domestication du dragon n'avait gagné de l'importance qu'entre-temps : signe, justement, d'un nouvel enjeu, la domination des communautés d'habitants (communiones dont le nouveau sens est, rappelons-le, condamné par Guibert de Nogent vers $1120^{114}$ ) ? Mais on remarquera également, dans le cas parisien, l'étonnante coïncidence structurelle entre l'affirmation de la mainmise ecclésiastique sur la ville par le biais du dragon des Rogations (et des sculptures de Notre-Dame ${ }^{115}$ ) d'une part et l'affirmation concurrente de la mainmise royale sur la ville par le biais de la lutte contre la boue.

En effet, c'est justement par le biais du réseau viaire que Philippe Auguste est censé avoir arraché la ville au monde du limon primitif et lui avoir permis d'accéder à la civilisation grâce au pavage des rues dans les années 1180, qui aurait valu à la ville de perdre son ancien nom de Lutèce, formé sur lutum, au profit de Paris, dérivé de Pâris, fils du roi de Troie (interprétation donnée par Rigord, moine de Saint-Denis, abbaye qui constitue la grande concurrente ecclésiale de la cathédrale). Et c'est rappelons-le Philippe Auguste qui met symboliquement la main sur la ville en la faisant entourer d'une muraille aux frais des bourgeois ${ }^{116}$. Avec ces décisions royales concernant les rues et les murail-

Stadtmauer als Grundlage städtischen Bewußtseins », dans Stadt und Krieg (25. Arbeitstagung in Böblingen, 1986), Sigmaringen, Thorbecke, 1989, p. 9-25; R. Gerber, Öffentliches Bauen im mittelalterlichen Bern. Verwaltungs- und finanzgeschichtliche Untersuchung über das Bauherrenamt der Stadt Bern 1300 bis 1550, Bern, Historischer Verein des Kantons Bern, 1994, p. 123-126. À propos du retour séparé de la collégiale Saint-Just le long du mur occidental de la ville de Lyon, P. Collomb, "Les processions des Rogations... », op. cit., p. 72, réduit de façon, me semble-t-il, indue ce trajet à un parcours de l'espace suburbain, donnant ainsi « de fait une dimension rurale aux Rogations ». Outre le fait que des champs cultivés se rencontraient intra-muros et qu'on devrait se demander pourquoi seule une branche secondaire des Rogations serait chargée de la ruralité, il me semble que c'est la volonté de trouver la bénédiction des champs qui conduit à une telle lecture (cf. ce qui a été dit supra, n. 65).

113. J. Le Goff, « Culture ecclésiastique... », op. cit., p. 271.

114. Guibert de Nogent, Autobiographie, éd. E.R. Labande, Paris, Belles-Lettres, 1981, p. 320 : communio autem novum ac pessimum nomen...

115. J. Le Goff, « Culture ecclésiastique... », op. cit., p. 263-266. A. Erlande-Brandenburg, " Notre-Dame de Paris », dans Les lieux de mémoire, III : Les France, t. 3, P. Nora dir., Paris, Gallimard, 1992, p. 359-401, ici p. 365-369, rappelle le contexte de la concurrence entre l'évêché et l'abbaye de Saint-Denis (cf. A. Lombard-Jourdan, «Montjoie et Saint-Denis ! »..., op. cit.) : la thématique de saint Marcel terrassant le dragon parait donc bien corrélée à une affirmation de la domination épiscopale à Paris.

116. Cf. J. Morsel, « Comment peut-on être Parisien ? Contribution à l'histoire de la genèse de la communauté parisienne au XIII ${ }^{\mathrm{e}}$ siècle », dans Religion et société urbaine..., op. cit., p. 363-381, ici p. 371-372, et « Appropriation communautaire du territoire, ou appropriation territoriale de la communauté ? Observations en guise de conclusion ", Hypothèses 2005. Travaux de l'École doc- 
les de Paris, ce qui est classiquement interprété comme de l'urbanisme avant la lettre pourrait n'être qu'une forme monumentale de réponse au mode processionnel d'affirmation cléricale sur la communauté d'habitants.

En l'état actuel (très insuffisant) de nos connaissances, les Rogations devraient par conséquent être sans doute considérées comme un rituel d'homogénéisation chrétienne de la communauté d'habitants, indissociable d'une volonté cléricale de s'affirmer sur son espace au moyen d'une déambulation articulée à l'espace chrétien, alors que le lien paroissial ne permet pas (en ville) ou pas encore (à la campagne) une telle affirmation. Prendre en compte en même temps la ville et le village permet de faire l'hypothèse que même lorsqu'au village les Rogations mobilisent les seuls paroissiens du lieu (à l'inverse du cas du Lyonnais), il ne s'agit pourtant pas d'une pratique stricto sensu paroissiale (même si elle l'est de facto) mais d'une forme de surimposition ecclésiale sur l'espace de la communauté d'habitants. Assimiler d'emblée les Rogations à une pratique paroissiale (elle-même conçue comme communautaire) ne peut que faire disparaitre le travail socio-spatial ${ }^{117}$ que nous avons appelé ici " paroissialisation ».

Ce faisant, les Rogations auraient permis que se mette en place une adéquation plus nette entre l'espace communautaire (en tant qu'espace d'appropriation collective de ressources locales, matérielles comme idéelles) et un espace chrétien local, non réductible par principe (même si c'est souvent le cas dans les campagnes) à la paroisse. Bref on aurait là affaire à un mode de production chrétienne de la communauté d'habitant, dont le succès se traduit entre autres par la folklorisation du dragon - c'est-à-dire l'appropriation par la population du signe de sa soumission ${ }^{118}$. C'est peut-être cette « communau-

torale d'histoire de l'Université Paris I Panthéon-Sorbonne, Paris, Publications de la Sorbonne, 2006, p. 89-104, ici p. 98.

117. À propos de cet adjectif, utilisé une demi-douzaine de fois dans cette contribution, il n'est certainement pas inutile de rappeler son caractère de truisme. La rubrique « Espace » dans Les mots de la géographie, dictionnaire critique, R. Brunet, R. Ferras, H. Théry dir., Paris, Reclus/La Documentation Française, $3^{\mathrm{e}}$ éd. 1993, p. 195, s'achève d'ailleurs ainsi : «l'espace n'est et ne peut être que social, ses lois ont des logiques sociales [...]. Il n'y a pas d' " espace naturel », mais des ensembles d'éléments physiques de l'espace géographique. Certes, l'absence des hommes n'empêcherait pas la Terre et l'Univers d'être ; mais ils n'existeraient pas comme "espaces". Il est donc tout à fait inutile d'employer l'adjectif "socio-spatial". »

118. Le registre de visites pastorales du diocèse d'Eichstätt montre un certain nombre de cas où la communauté impose au curé la procession dans les champs ou la célébration des saints protecteurs (à Oberhaunstadt, Hofstetten, Walting, Schelldorf, Tauernfeld, Eichenhofen, Fahr- 
nautarisation » locale des Rogations qui aurait rendu nécessaire la mise en place, à la fin du XIII ${ }^{\mathrm{e}}$ siècle, d'une nouvelle procession générale, centrée sur le corps du christ et donc sur le phénomène de production (multilocale) de l'ecclesia dans son ensemble ${ }^{119}$.

\section{Conclusion}

Au-delà des spécificités propres aux lieux, aux échelles d'analyse et aux processions considérées dans les pages qui précèdent, il nous semble clair que le processus d'encellulement et de formation de communautés d'habitants a certainement reposé en partie sur l'organisation de systèmes processionnels, qui font de l'espace non pas un cadre amorphe mais un facteur par lequel advient ce nouveau mode d'encadrement social qu'est la communauté d'habitants. Ceci ne signifie évidemment pas l'apparition alors des processions mais, en rupture avec la tentation « continuationniste ", la dotation de cette pratique d'un sens social nouveau, qui est certainement allé de pair avec un changement de références spatiales (les paroisses), avec la production d'instruments normatifs (les manuels de liturgie) et avec la mise en place d'un imaginaire collectif (les dragons).

Ce sens social nouveau peut être défini, en une sorte de paradoxe, comme un mode de déplacement qui fixe les populations au sol - fixation au sol qui

rieden, Offenhausen, Hüssingen, Lellenfeld). Plutôt que d'y voir une forme de croyance populaire plus ou moins folklorique et issue de vieilles pratiques païennes, j'aurais plutôt tendance à considérer qu'il s'agit là de signes de ce que les populations ont remarquablement intégré l'idée selon laquelle le clergé est le seul intermédiaire valable entre elles et dieu, donc qu'elles se sont donc bien approprié les discours cléricaux. L. Kuchenbuch avait montré supra comment les processions obligatoires (domaniales) étaient devenues non obligatoires ; désormais, ce sont les paroissiens eux-mêmes qui les demandent : le rapport s'est complètement inversé.

119. Un facteur de développement du la Fête-Dieu que l'on tend en général à négliger est que contrairement aux reliques (à la base des Rogations), que n'importe qui pouvait détenir chez soi et qui donc n'étaient pas un élément de focalisation exclusive sur les églises, l'hostie était censée ne se trouver que dans les églises (ou dans les ostensoirs portés en procession). Plutôt que d'analyser le succès de la Fête-Dieu en simples termes de piété populaire (le désir de voir l'hostie), il faudrait prendre en compte les effets spatiaux spécifiques de cette procession. Bien que C. Zika, "Hosts, Processions... ", op. cit., tende à s'enfermer dans la problématique de la religiosité et des croyances populaires, dans lesquelles l'hostie est conçue comme une powerful relic (p. 48), il rappelle le caractère spécifique de l'hostie en tant qu'elle est directement liée au pouvoir du clergé (p. 60). Les efforts de divers théologiens de la fin du Xve et du début du $\mathrm{XVI}^{\mathrm{e}}$ siècle de définir des modes d'accès légitime à l'hostie (notamment à l'encontre de pèlerinages sauvages vers les hosties saignantes, comme celui de Wilsnack, en Saxe, en 1475) ne peuvent ainsi être réduits à de purs enjeux théologiques, mais prennent place dans une general attack on sacred mobility qui vise à renforcer la force d'attraction des églises paroissiales (p. 62). 
n'a justement rien d'une opération brutale (elle est d'ailleurs corrélative de la grande vague d'affranchissements et de disparition du servage) mais réside dans l'inculcation de l'esprit de clocher et de l'identification au lieu d'habitation. Aller à la messe ou aux processions devient par conséquent équivalent au fait d'aller cultiver ses parcelles : deux modalités d'intégration aux communautés d'habitants.

\author{
Ludolf KUCHENBUCH \\ Fernuniversität Hagen \\ Joseph MORSEL
}

Université Paris 1 Panthéon-Sorbonne / Lamop

Dieter SCHELER

Université de Bochum 


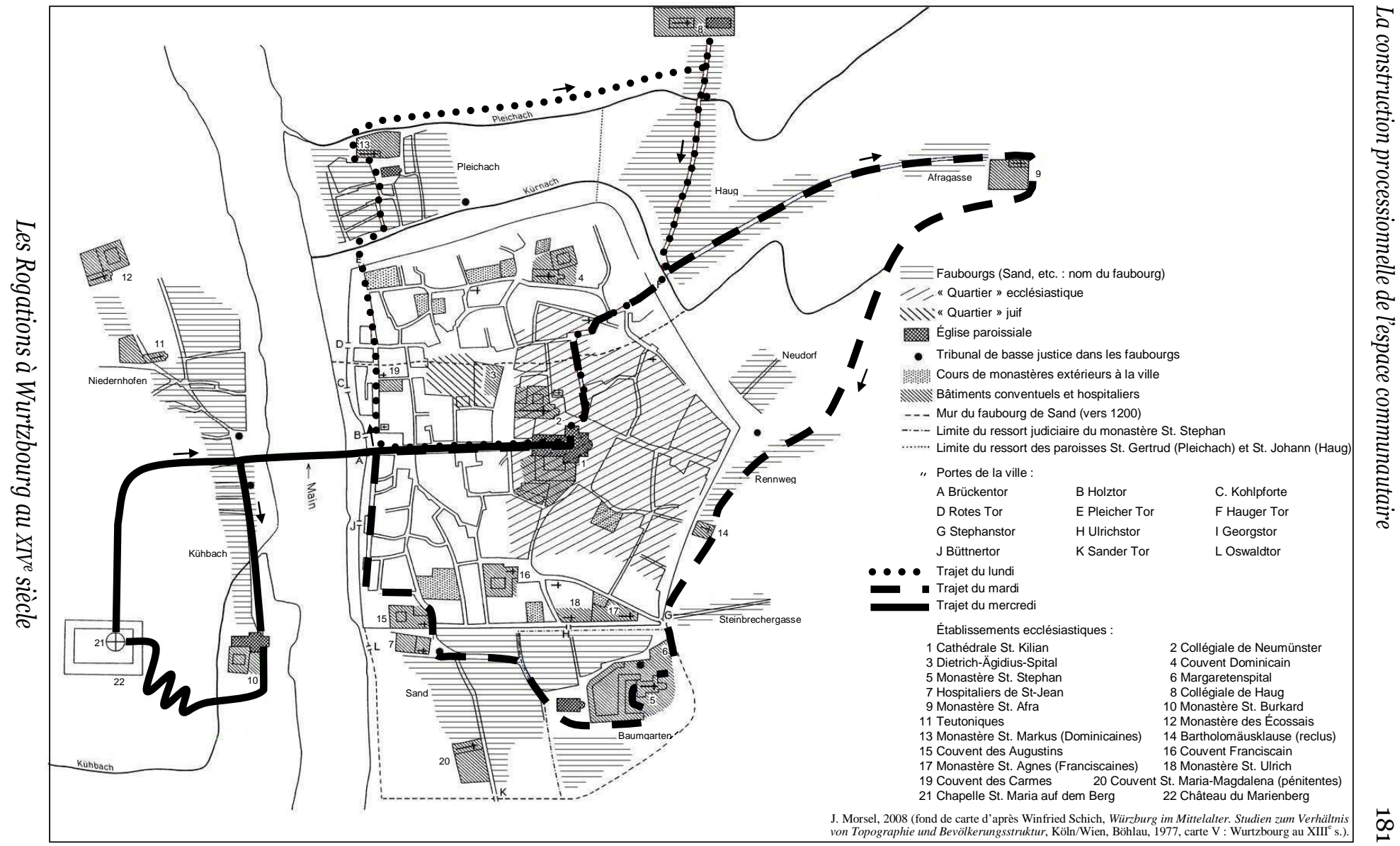



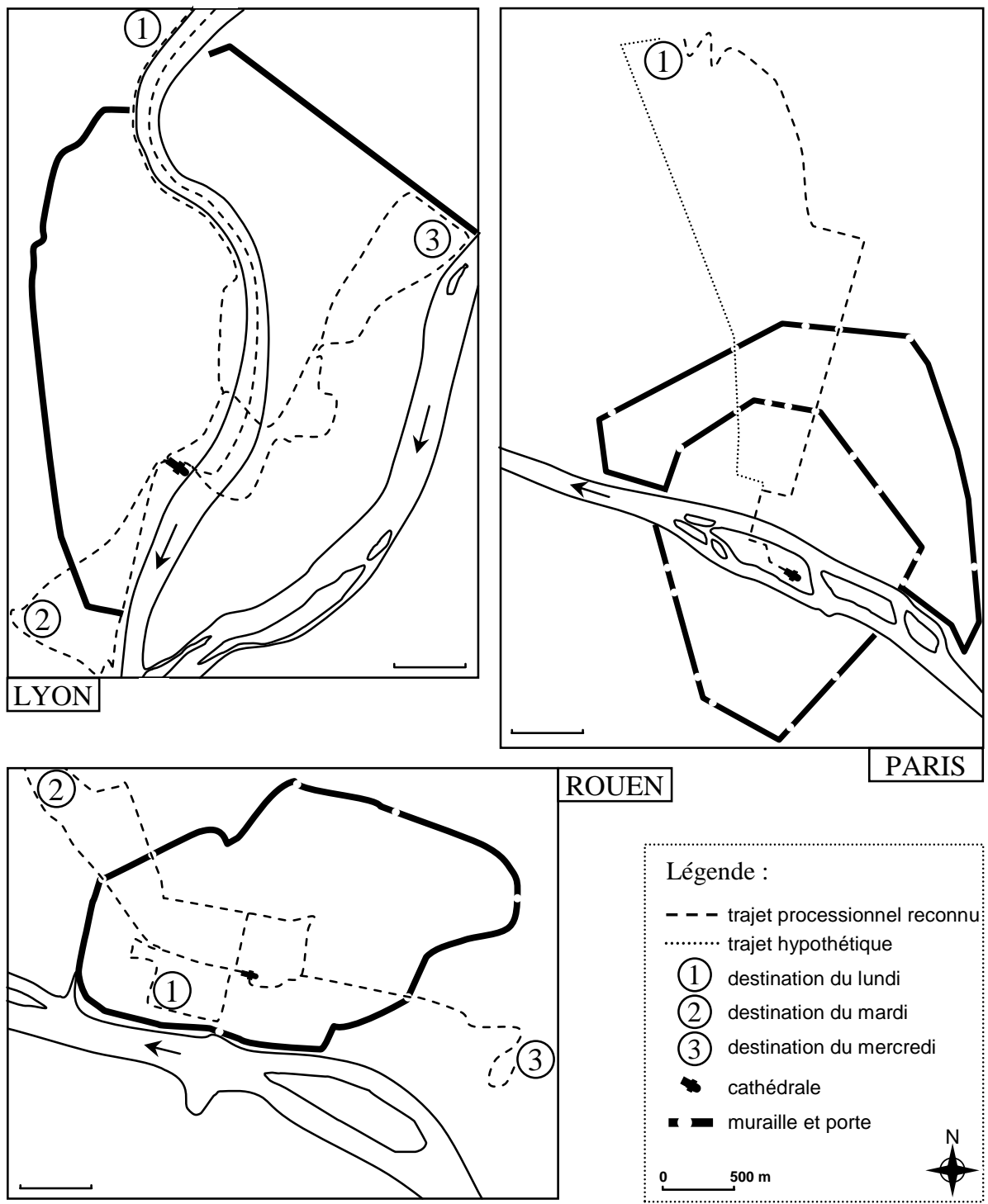

ROUEN
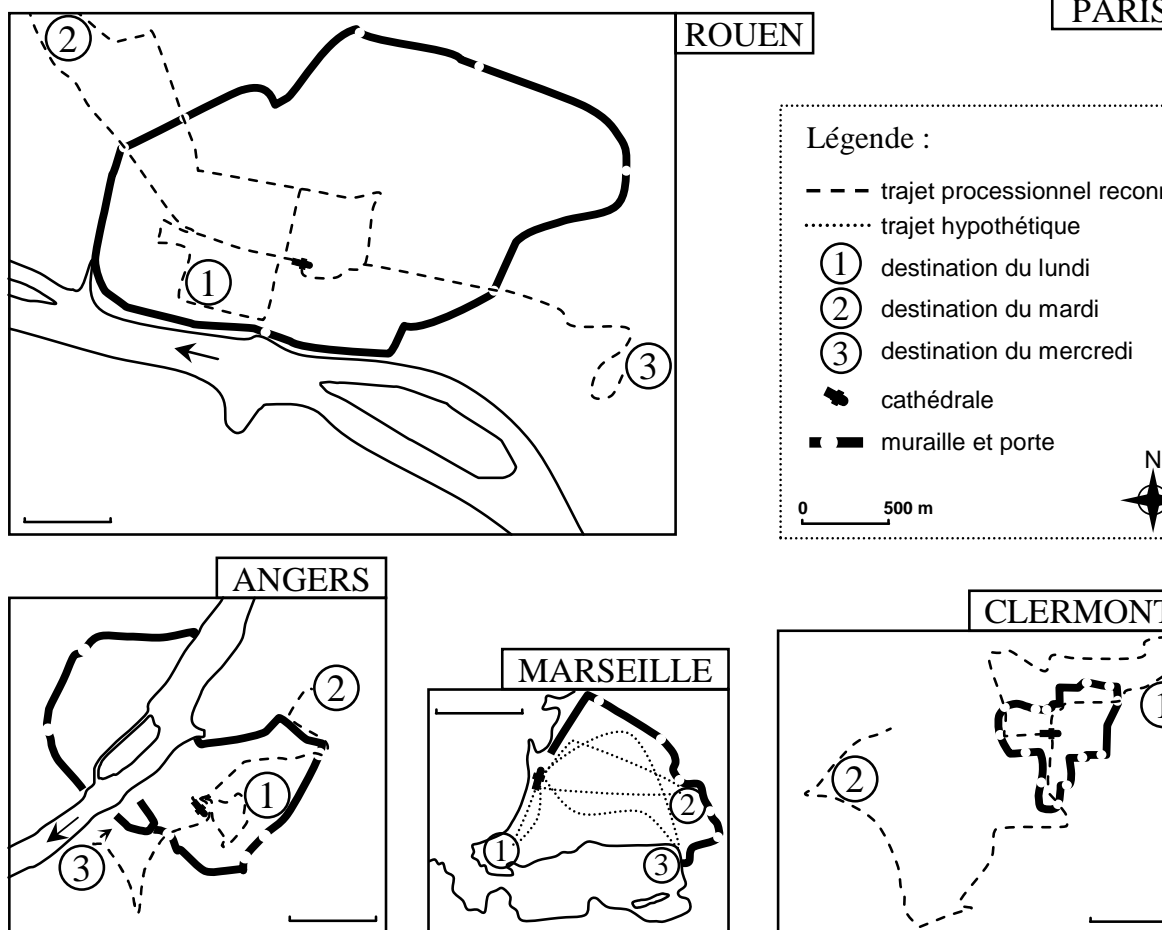

Légende :

- - - trajet processionnel reconnu

......... trajet hypothétique

(1) destination du lundi

(2) destination du mardi

(3) destination du mercredi

- cathédrale

- muraille et porte

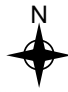

Itinéraires schématiques des Rogations dans quelques villes françaises médiévales 